\title{
A Parameterization with a Constrained Potential Energy Conversion Rate of Vertical Mixing Due to Langmuir Turbulence
}

\author{
BRANDON G. REICHL ${ }^{\mathrm{a}}$ \\ Program in Atmospheric and Oceanic Science, Princeton University, Princeton, New Jersey \\ QING Li ${ }^{\mathrm{b}}$ \\ Department of Earth, Environmental, and Planetary Sciences, Brown University, Providence, Rhode Island
}

(Manuscript received 5 December 2018, in final form 19 August 2019)

\begin{abstract}
In this study we develop a new parameterization for turbulent mixing in the ocean surface boundary layer (OSBL), including the effect of Langmuir turbulence. This new parameterization builds on a recent study (Reichl and Hallberg 2018, hereafter RH18), which predicts the available energy for turbulent mixing against stable stratification driven by shear and convective turbulence. To investigate the role of Langmuir turbulence in the framework of RH18, we utilize data from a suite of previously published large-eddy simulation (LES) experiments ( $\mathrm{Li}$ and Fox-Kemper 2017, hereafter LF17) with and without Langmuir turbulence under different idealized forcing conditions. We find that the parameterization of RH18 is able to reproduce the mixing simulated by the LES in the non-Langmuir cases, but not the Langmuir cases. We therefore investigate the enhancement of the integrated vertical buoyancy flux within the entrainment layer in the presence of Langmuir turbulence using the LES data. An additional factor is introduced in the RH18 framework to capture the enhanced mixing due to Langmuir turbulence. This additional factor depends on the surface-layer averaged Langmuir number with a reduction in the presence of destabilizing surface buoyancy fluxes. It is demonstrated that including this factor within the RH18 OSBL turbulent mixing parameterization framework captures the simulated effect of Langmuir turbulence in the LES, which can be used for simulating the effect of Langmuir turbulence in climate simulations. This new parameterization is compared to the KPP-based Langmuir entrainment parameterization introduced by LF17, and differences are explored in detail.
\end{abstract}

\section{Introduction}

Turbulence in the ocean surface boundary layer (OSBL) leads to vigorous mixing of properties from the atmosphere (or cryosphere) above and ocean interior below. The OSBL is therefore fundamental to net exchange of heat, gases, and other quantities between the atmosphere and the ocean interior. In large-scale ocean circulation models, such as for simulating Earth's climate, the OSBL turbulent mixing is parameterized. These turbulent mixing parameterizations are

\footnotetext{
${ }^{\text {a }}$ Current affiliation: NOAA Geophysical Fluid Dynamics Laboratory, Princeton, New Jersey.

${ }^{\mathrm{b}}$ Current affiliation: Fluid Dynamics and Solid Mechanics, Los Alamos National Laboratory, Los Alamos, New Mexico.
}

Corresponding author: Brandon G. Reichl, brandon.reichl@ noaa.gov formulated with insight about the characteristics of OSBL turbulence, which depend on the generation mechanism of the turbulence.

In this study we consider three mechanisms that drive OSBL turbulence: the Eulerian current shear (shear turbulence), gravitational instabilities (convective turbulence), and surface waves through the Stokes-drift shear (Langmuir turbulence). The primary focus of this study is to extend the energetics-based planetary boundary layer (ePBL) parameterization for shear and convective turbulence of Reichl and Hallberg (2018, hereafter RH18) to include the enhanced turbulent mixing due to Langmuir turbulence. This will be accomplished through analysis of the energetic implications of the interaction of the three types of turbulence in setting the vertically integrated entrainment buoyancy flux within the OSBL. Breaking waves are also critical to the generation and dissipation of OSBL turbulence, especially near the ocean surface, but are not 
considered for this study (see Sullivan et al. 2007; McWilliams et al. 2012).

\section{a. Langmuir turbulence in the $O S B L$}

A property of ocean surface wave motion is a vertically sheared mean Lagrangian current, known as the Stokes drift $\tilde{u}_{i}$ (see Van Den Bremer and Breivik 2017, and references therein). ${ }^{1}$ The surface magnitude of Stokes drift $\tilde{u}_{i 0}$ is set by the wave amplitude and its exponential decay scale with depth by the wavelength $\lambda\left[\tilde{u}_{i}=\tilde{u}_{i 0} \exp (2 k z)\right.$, where $k=2 \pi / \lambda$ is the wavenumber]. Superposition of waves of the full directional wavenumber wave spectra determines the net Stokes drift and its direction. The Stokes drift modifies the characteristics of turbulence relative to an environment without surface waves as its vertical shear plays a distinct role in turbulence generation from Eulerian current mean shear (e.g., Teixeira and Belcher 2002; Grant and Belcher 2009). The modified turbulence due to Stokes drift is known as Langmuir turbulence (following McWilliams et al. 1997), as the mechanism was proposed as a result of efforts to understand Langmuir circulations in the ocean (following Craik and Leibovich 1976).

Ocean climate simulations with and without parameterization of the effect of Langmuir turbulence suggest it plays a significant role in global OSBL processes (see Belcher et al. 2012; D'Asaro 2014; Fan and Griffies 2014; Li et al. 2016; Noh et al. 2016). Surface waves also play a critical role in OSBL near-surface mixing and air-sea fluxes through wave breaking (e.g., Melville 1996; Pizzo et al. 2016). Surface wave breaking in the OSBL primarily enhances the turbulence from traditional (law of the wall) scaling over a layer confined near to the surface (Agrawal et al. 1992; Anis and Moum 1995; Gerbi et al. 2009; Thomson et al. 2016). Langmuir turbulence, however, modifies turbulence properties and entrainment rates throughout the OSBL based on numerous descriptive studies based on field campaigns (e.g., Weller and Price 1988; Smith 1992; Kukulka et al. 2009; Hoecker-Martínez et al. 2016) and modeling studies (e.g., Skyllingstad and Denbo 1995; McWilliams et al. 1997; Noh et al. 2004; Li et al. 2005; Polton and Belcher 2007; Harcourt and D'Asaro 2008; Kukulka et al. 2010; Van Roekel et al. 2012). Langmuir turbulence is hypothesized to be the primary wave-driven mechanism modifying entrainment at the base of the OSBL (following Sullivan et al. 2007; McWilliams et al. 2012).

\footnotetext{
${ }^{1}$ The convention $i=1,2$ corresponds to horizontal directional indices and $w=u_{3}$ is used interchangeably for the vertical index.
}

\section{b. Parameterizations for Langmuir turbulence}

Parameterizations including the effect of Langmuir turbulence have included modifications to the $K$-profile parameterization (KPP; Large et al. 1994; McWilliams and Sullivan 2000; Smyth et al. 2002; Sinha et al. 2015; Reichl et al. 2016; Li et al. 2016; Li and Fox-Kemper 2017) and to turbulent kinetic energy (TKE) closure (D'Alessio et al. 1998; Axell 2002; Kantha and Clayson 2004; Harcourt 2013, 2015; Noh et al. 2016). In the KPP approach, the turbulent velocity scale is typically enhanced using LES-derived relationships between the turbulent vertical velocity variance ${ }^{2} \overline{w^{\prime} w^{\prime}}$ and the Langmuir number (La), which generally represents the relative importance of wind-driven to Langmuirdriven turbulence. However, these relationships are inadequate to use for the total enhancement to the turbulent mixing coefficient, since the turbulent mixing length is also modified (e.g., Reichl et al. 2016). The enhancement of the velocity scale also differs from the enhancement to entrainment in KPP (see McWilliams et al. 2014), motivating at least two modifications of the KPP boundary layer depth calculation to account for Langmuir turbulence for climate (Li and Fox-Kemper 2017) and hurricane conditions (Reichl et al. 2016). TKE closure includes the effects of Langmuir turbulence through a Stokes production term added to the TKE budget (D'Alessio et al. 1998; Axell 2002; Kantha and Clayson 2004). In TKE closure, the role of Langmuir turbulence is not fully captured through its effect on the energetics, so further modifications have been proposed via length scale enhancement in one-equation closure (e.g., Noh et al. 2016) and via the stability functions in modified second-moment closure (Harcourt 2013, 2015).

A nondimensional parameter commonly employed to formulate Langmuir turbulence parameterizations is the Langmuir number, which comprises a ratio of wind-friction speed $u_{*}$ to Stokes drift. The specific form of the Langmuir number appropriate for parameterizing Langmuir turbulence in KPP has been investigated several times since the turbulent Langmuir number was introduced in McWilliams et al. (1997):

$$
\mathrm{La}_{t}=\left(\frac{u_{*}}{\left|\tilde{u}_{i 0}\right|}\right)^{1 / 2},
$$

and subsequently used to parameterize the effects of Langmuir turbulence in KPP by McWilliams and Sullivan (2000).

\footnotetext{
${ }^{2}$ The averaging operator is not formally defined here, except to note that $\overline{x^{\prime}}=0$, where $x$ is an arbitrary parameter.
} 
Harcourt and D'Asaro (2008) found that a surface layer averaged Langmuir number ( $\left.\mathrm{La}_{\mathrm{SL}}\right)$ more accurately captures the effect of Langmuir turbulence on long-lasting, bulk turbulence properties (specifically the vertical average of vertical velocity variance over the boundary layer $\left\langle\overline{w^{\prime} w^{\prime}}\right\rangle_{\mathrm{bl}}$, where the brackets denote vertical mean) by considering the vertical decay of Stokes drift relative to the boundary layer depth $H_{\mathrm{b} \text { l }}$ :

$$
\mathrm{La}_{\mathrm{SL}}=\left[\frac{u_{*}}{\frac{1}{0.2 H_{\mathrm{bl}}} \int_{-0.2 H_{\mathrm{bl}}}^{0}\left|\tilde{u}_{i}-\tilde{u}_{i}\left(z=-H_{\mathrm{bl}}\right)\right| d z}\right]^{1 / 2},
$$

where $\tilde{u}_{i}\left(z=-H_{\mathrm{bl}}\right)$ accounts for any significant Stokes drift near the base of the OSBL that does not contribute to the turbulent mixing in the OSBL. A projected surface-layer averaged Langmuir number $\left(\mathrm{La}_{\mathrm{SL} p}\right)$ was later introduced by Van Roekel et al. (2012) to include the effect of misalignment between Langmuir cells and the wind stress. Reichl et al. (2016) subsequently found that $\mathrm{La}_{\mathrm{SL} p}$ (in a slightly modified form) was preferred for parameterization of Langmuir turbulence in KPP for hurricane conditions. A KPP parameterization using $\mathrm{La}_{\mathrm{SL} p}$ also yielded the best prediction of global OSBL mixing in a comparative climate study by Li et al. (2016). More general definitions of the Langmuir number, such as based on the spectrally filtered surface Stokes drift (Kukulka and Harcourt 2017), have also been proposed. It is not known if a single form of the Langmuir number is preferred for parameterization of all aspects of Langmuir turbulence. We therefore consider possible forms of the Langmuir number in this study.

\section{c. Outline}

The primary goal of this study is to extend the RH18 parameterization to account for additional mixing due to Langmuir turbulence. We focus specifically on the effect of Langmuir turbulence on the bulk OSBL properties through the enhancement to the integrated entrainment buoyancy flux. In section 2 , we introduce the primary datasets used for this study and the relevant numerical models. In section 3, we then describe relevant quantities and modifications needed to parameterize the turbulent mixing and Langmuir turbulence following the RH18 approach. In section 4, we demonstrate the application of these results in onedimensional model simulations and compare our methods directly to results obtained using the approach of Li and Fox-Kemper (2017). The symbols and acronyms used within this study are listed for convenience in Tables 1 and 2.
TABLE 1. List of symbols used for this study.

\begin{tabular}{|c|c|c|}
\hline Symbol & MKS units & Description \\
\hline$a$ & None & $\begin{array}{l}\text { Linear decay rate in mechanical velocity } \\
\text { scale }\end{array}$ \\
\hline$\alpha_{x}$ & None & $A$ empirical coefficients \\
\hline$A$ & None & $\begin{array}{l}\text { Entrainment-peak/entrainment-base } \\
\text { relation coefficient }\end{array}$ \\
\hline$b$ & $\mathrm{~ms} \mathrm{~s}^{-2}$ & Buoyancy \\
\hline$B_{f}$ & $\mathrm{~m}^{2} \mathrm{~s}^{-3}$ & Surface buoyancy flux $\left(-\overline{w^{\prime} b_{0}^{\prime}}\right)$ \\
\hline$B_{\text {Frac }}$ & None & Fraction of total production by buoyancy \\
\hline$C_{K}$ & None & Turbulent stability coefficient \\
\hline$c_{\mu}^{0}$ & None & Turbulent viscosity empirical coefficient \\
\hline$c_{N x}$ & None & $m_{*_{N}}$ empirical coefficients \\
\hline$c_{S x}$ & None & $m_{* s}$ empirical coefficients \\
\hline$c_{\Psi}$ & None & Mechanical reduction coefficient \\
\hline$\varepsilon_{\mathrm{bl}}$ & $\mathrm{m}^{3} \mathrm{~s}^{-3}$ & Integrated dissipation of TKE \\
\hline$H_{e}$ & $\mathrm{~m}$ & Entrainment layer thickness \\
\hline$H_{\mathrm{bl}}$ & $\mathrm{m}$ & Boundary layer thickness \\
\hline$f$ & $\mathrm{~s}^{-1}$ & Coriolis frequency \\
\hline$\gamma$ & None & Length-scale shape function exponent \\
\hline$G_{\mathrm{bl}}$ & $\mathrm{m}^{3} \mathrm{~s}^{-3}$ & $\begin{array}{l}\text { Integrated convective buoyancy } \\
\text { production }\end{array}$ \\
\hline$\kappa$ & None & von Kaŕmań constant \\
\hline$k$ & $\mathrm{~m}^{-1}$ & Surface gravity wave wavenumber \\
\hline$L_{O}$ & $\mathrm{~m}$ & Obukhov length \\
\hline $\mathrm{La}_{t}$ & None & Turbulent Langmuir number \\
\hline $\mathrm{La}_{\mathrm{SL}}$ & None & Surface-layer averaged Langmuir number \\
\hline$\lambda$ & $\mathrm{m}$ & Surface gravity wave wavelength \\
\hline$K$ & $\mathrm{~m}^{2} \mathrm{~s}^{-1}$ & Turbulent viscosity \\
\hline$L$ & $\mathrm{~m}$ & Turbulent length scale \\
\hline$m_{*}$ & None & $\begin{array}{l}\text { Mechanical entrainment proportionality } \\
\text { coefficient }\end{array}$ \\
\hline$m_{*_{N}}$ & None & $\begin{array}{l}\text { See } m_{*} \text {, contribution due to neutral } \\
\text { surface flux }\end{array}$ \\
\hline$m * s$ & None & $\begin{array}{l}\text { See } m_{*} \text {, contribution due to stabilizing } \\
\text { surface flux }\end{array}$ \\
\hline$M_{e}$ & $\mathrm{~m}^{3} \mathrm{~s}^{-3}$ & Integrated entrainment buoyancy flux \\
\hline$n_{*}$ & None & $\begin{array}{l}\text { Convective entrainment proportionality } \\
\text { coefficient }\end{array}$ \\
\hline$p$ & $\mathrm{~m}^{2} \mathrm{~s}^{-2}$ & Kinematic pressure \\
\hline$u_{*}$ & $\mathrm{~ms}^{-1}$ & Air-sea interface friction velocity \\
\hline$u_{i}$ & $\mathrm{~m} \mathrm{~s}^{-1}$ & Horizontal components of velocity \\
\hline$\tilde{u}$ & $\mathrm{~m} \mathrm{~s}^{-1}$ & Stokes drift \\
\hline$\Psi$ & None & Mechanical production reduction factor \\
\hline$v_{*}$ & $\mathrm{~m} \mathrm{~s}^{-1}$ & $\begin{array}{l}\text { Mechanical component of turbulent } \\
\text { velocity }\end{array}$ \\
\hline$w$ & $\mathrm{~m} \mathrm{~s}^{-1}$ & Vertical component of velocity \\
\hline$w_{*}$ & $\mathrm{~m} \mathrm{~s}^{-1}$ & $\begin{array}{l}\text { Convective component of turbulent } \\
\text { velocity }\end{array}$ \\
\hline$w_{t}$ & $\mathrm{~m} \mathrm{~s}^{-1}$ & Turbulent velocity scale \\
\hline$\overline{w^{\prime} b_{e}^{\prime}}$ & $\mathrm{m}^{2} \mathrm{~s}^{-3}$ & Peak entrainment buoyancy flux \\
\hline$z_{0}$ & $\mathrm{~m}$ & Surface roughness length \\
\hline$Z_{\mathrm{bl}}$ & $\mathrm{m}$ & Depth of base of boundary layer \\
\hline$Z_{e T}$ & $\mathrm{~m}$ & Depth of top of entrainment layer \\
\hline$Z_{e B}$ & $\mathrm{~m}$ & Depth of bottom of entrainment layer \\
\hline$Z_{B}$ & $\mathrm{~m}$ & Depth of base of LES domain \\
\hline
\end{tabular}


TABLE 2. List of acronyms used for this study.

\begin{tabular}{ll}
\hline \hline Acronym & \multicolumn{1}{c}{ Description } \\
\hline ePBL & Energetic planetary boundary layer \\
KPP & K-profile parameterization \\
LES & Large-eddy simulation \\
LF17 & Li and Fox-Kemper (2017) \\
OSBL & Ocean surface boundary layer \\
PE & Potential energy \\
RH18 & Reichl and Hallberg (2018) \\
SST & Sea surface temperature \\
TKE & Turbulent kinetic energy \\
\hline
\end{tabular}

\section{Methods}

This study investigates the contribution of Langmuir turbulence to turbulent mixing in mixed convective and shear-turbulence regimes. There are numerous difficulties that prevent precise investigation of this process from in situ or laboratory settings. These difficulties include reproducing the necessary range of scales and phenomena in the laboratory and obtaining the accuracy, quantity, and type of measurements needed to carefully diagnose and isolate OSBL turbulent processes in the field. Therefore, we utilize idealized numerical simulations for the present investigation, though future validation against field data is critical.

\section{a. LES and one-dimensional MOM6 experiments}

The primary numerical simulations used for this study are the large-eddy simulations (LES) previously presented in Li and Fox-Kemper (2017, hereafter LF17). We briefly review their LES experiments here and refer the reader to the original citation for details. LF17 simulates the wave-averaged Craik-Leibovich equations for a wide range of external forcings including wind (5-10 $\mathrm{m} \mathrm{s}^{-1}$ ), wave (broadband empirical spectra of varying wave age), and convective (from -5 to $-500 \mathrm{~W} \mathrm{~m}^{-2}$ ) conditions. To investigate the role of varying Langmuir turbulence we obtain simulations from LF17 employing Stokes drift calculated from the Donelan et al. (1985) empirical wave spectra with up to four different wave ages. This set of experiments covers typical wind, wave, and destabilizing buoyancy flux conditions in the global ocean in dimensionless space (see LF17).

We conduct three additional simulations using the identical model approach as LF17. The first simulation varies the Coriolis parameter $f$, since $\mathrm{RH} 18$ finds that $u_{*} / H_{\mathrm{b} \mid}|f|$ is an important nondimensional parameter for predicting the integrated vertical buoyancy flux. The other simulations include surface sensible heating ( 25 and $50 \mathrm{~W} \mathrm{~m}^{-2}$ ), since a stabilizing flux restricts the turbulence and shoals the Obukhov depth $L_{O}$. The LES experiments considered here are grouped and labeled
TABLE 3. List of LES experiments. Note that for experiments 318 the latitude is set to $45^{\circ}$, while for experiment 19 (marked with an asterisk) the latitude was set to $22.5^{\circ}$.

\begin{tabular}{cccc}
\hline \hline Experiment & $\begin{array}{c}\text { Surface heat } \\
\text { flux }\left(\mathrm{W} \mathrm{m}^{-2}\right)\end{array}$ & $\begin{array}{c}\text { Wind speed } \\
\left(\mathrm{m} \mathrm{s}^{-1}\right)\end{array}$ & Wave ages \\
\hline 3 & -5 & 5 & $0,0.6,0.8,1.0,1.2$ \\
4 & -5 & 8 & $0,0.6,0.8,1.0,1.2$ \\
5 & -5 & 10 & $0,0.6,0.8,1.0,1.2$ \\
6 & -10 & 5 & $0,0.8,1.2$ \\
7 & -25 & 5 & $0,0.8,1.2$ \\
8 & -25 & 8 & $0,0.8,1.2$ \\
9 & -25 & 10 & $0,0.8,1.2$ \\
10 & -50 & 5 & $0,0.8,1.2$ \\
11 & -50 & 8 & $0,0.8,1.2$ \\
12 & -50 & 10 & $0,0.8,1.2$ \\
13 & -100 & 5 & $0,0.8,1.2$ \\
14 & -200 & 5 & $0,0.8,1.2$ \\
15 & -300 & 5 & $0,0.8,1.2$ \\
16 & -500 & 5 & $0,0.8,1.2$ \\
17 & 25 & 5 & $0,0.8,1.2$ \\
18 & 50 & 5 & $0,0.8,1.2$ \\
$19 *$ & -5 & 5 & $0,0.6,0.8,1.0,1.2$ \\
\hline
\end{tabular}

by ID numbers for quick reference and the full list is given in Table 3. Using these LES data, the effect of Langmuir turbulence within the ePBL/RH18 framework is assessed and ePBL is modified accordingly.

A one-dimensional version of the Modular Ocean Model 6 (MOM6; Adcroft et al. 2018) is used here to drive the new parameterization and validate it against the LES. These one-dimensional simulations are initialized from the same temperature profiles and forced by similar surface forcing as the LES experiments. In addition, MOM6 can use KPP (Large et al. 1994; Van Roekel et al. 2018) via the Community Vertical Mixing (CVMix) package (see github.com/CVMix). This allows the modified ePBL parameterization to be compared directly to experiments with KPP-based Langmuir turbulence parameterization (such as LF17).

\section{b. The ePBL parameterization}

The ePBL parameterization of OSBL turbulent mixing is described in detail by RH18 and briefly reviewed here. The vertical turbulent buoyancy flux $\overline{w^{\prime} b^{\prime}}$ represents the average correlation of fluctuations in vertical velocity $w^{\prime}$ and buoyancy $b^{\prime}$. This flux describes the rate of conversion between turbulent kinetic energy (TKE) and potential energy (PE). The vertical integral of this flux (hereafter $M_{e}$ ) over the entrainment layer is given by

$$
M_{e}=\int_{Z_{e B}}^{Z_{e T}} \overline{w^{\prime} b^{\prime}} d z
$$

where $Z_{e T}$ and $Z_{e B}$ are the top and bottom depths of the entrainment zone. The entrainment zone, with thickness 
$H_{e}=Z_{e T}-Z_{e B}$, is defined to encompass the region in the OSBL where the buoyancy flux is negative, $\overline{w^{\prime} b^{\prime}}<0$. In this region, the turbulent buoyancy flux mixes denser water upward (against gravity), thus driving an integrated PE change in the water column. The ePBL is formulated such that the total PE increase due to entrainment by the turbulent buoyancy flux is constrained via a parameterized value for $M_{e}$.

The turbulent fluxes, such as the turbulent buoyancy flux, are parameterized in ePBL using a mixing coefficient $K$ :

$$
\overline{w^{\prime} b^{\prime}}=-K \partial_{z} \bar{b} .
$$

Equation (4) is used in Eq. (3) to relate $M_{e}$ with the boundary layer depth, $H_{\mathrm{bl}}=-Z_{e B}$, and $K$ via an implicit, iterative integral constraint:

$$
M_{e}=\int_{-H_{\mathrm{bl}}}^{0} \min \left(0,-K \partial_{z} \bar{b}\right) d z,
$$

where the minimum function is employed to distinguish the entrainment region from the convecting region. The mixing coefficient $K$ is parameterized from the product of a turbulent velocity $w_{t}$ and turbulent length $L$ :

$$
K(z)=C_{K} w_{t}(z) L(z)
$$

where $C_{K}$ is a constant, empirical coefficient.

Empirical parameterizations for $w_{t}$ and $L$ are given in RH18. For $w_{t}$, turbulent velocity sources are decomposed into convective $w_{*}$ and mechanical $v_{*}$ contributions:

$$
w_{t}(z)=w_{*}(z)+v_{*}(z) .
$$

The convective contribution is found from the integral of the buoyancy flux to depth $z$ via

$$
w_{*}(z)=C_{w_{*}}\left(\int_{z}^{0} \overline{w^{\prime} b^{\prime}} d z\right)^{1 / 3},
$$

while the mechanical contribution is parameterized from the surface friction velocity as

$$
v_{*}(z)=\left(c_{\mu}^{0}\right)^{1 / 3} u_{*}\left[1-a \min \left(1, \frac{|z|}{H_{\mathrm{bl}}}\right)\right],
$$

where $C_{w *}$ and $c_{\mu}^{0}$ are empirical coefficients and $a$ is a fixed vertical decay scale. The length scale is found from wall theory as

$$
L(z)=\left(z_{0}+|z|\right)\left(\frac{H_{\mathrm{bl}}-|z|}{H_{\mathrm{bl}}}\right)^{\gamma},
$$

where $z_{0}>0$ is an empirical surface roughness length. The exponent $\gamma$ in this length scale relation determines the vertical shape of $L$ (and hence $K$ ), with $\gamma=$ 1 having a law-of-the-wall profile at both the surface and the boundary layer depth and $\gamma=2$ approximating the KPP cubic shape function. This simplified approach for parameterizing $K$ is motivated for implementing in present generation climate model configurations (see RH18) as it accommodates constraints in vertical grids (typically less than 100 vertical levels to resolve the full ocean) and long time steps (coupling time steps of order $1 \mathrm{~h}$ ). Advancing computational capabilities may alleviate these constraints to an extent, though optimal resource allocation between model complexity and model ensemble size remains debated (see Hewitt et al. 2017). Additionally, efforts such as subcycling and using alternative hardware (specifically graphics processing units) to accommodate more complex $K$ parameterization approaches [e.g., TKE closure such as Harcourt (2015)] in climate models are a topic of present research.

The equations used to form the mixing coefficient by ePBL are closed via parameterizations for $M_{e}$. In section 3 we discuss this approach, including the existing parameterizations supplied by RH18 and the modifications needed for including Langmuir turbulence.

\section{c. KPP with LF17 Langmuir entrainment}

We refer the reader to Large et al. (1994) and Van Roekel et al. (2018) for comprehensive descriptions of KPP and its algorithms. Here we briefly review the main points of KPP as pertaining to the LF17 Langmuir turbulence modification for comparison with the results of this study.

In KPP the mixing coefficient formulation within the surface boundary layer is written

$$
K_{\mathrm{KPP}}(\sigma)=G(\sigma) H_{\mathrm{bl}} w_{\mathrm{KPP}},
$$

where $G(\sigma)$ is a nondimensional shape function varying in the vertical between zero and unity based on $\sigma=$ $|z| / H_{\mathrm{bl}}$. Equation (11) is effectively equivalent to Eq. (6) if we assume $L \approx G(\sigma) H_{\mathrm{bl}}$ and the nondimensional coefficient is absorbed into the length and/or velocity scale. The definition of $w_{\mathrm{KPP}}$ and $w_{t}$ can vary depending on the application, and these differences and their respective enhancement due to Langmuir turbulence are not discussed in detail here (see Van Roekel et al. 2012; Reichl et al. 2016; LF17; RH18).

A key difference between ePBL and KPP is due to the definition of the length scale as it pertains to the boundary layer thickness. In KPP, the boundary layer depth $H_{\mathrm{bl}}$ is diagnosed from the depth where vertical profile of the bulk Richardson number exceeds a critical value: 

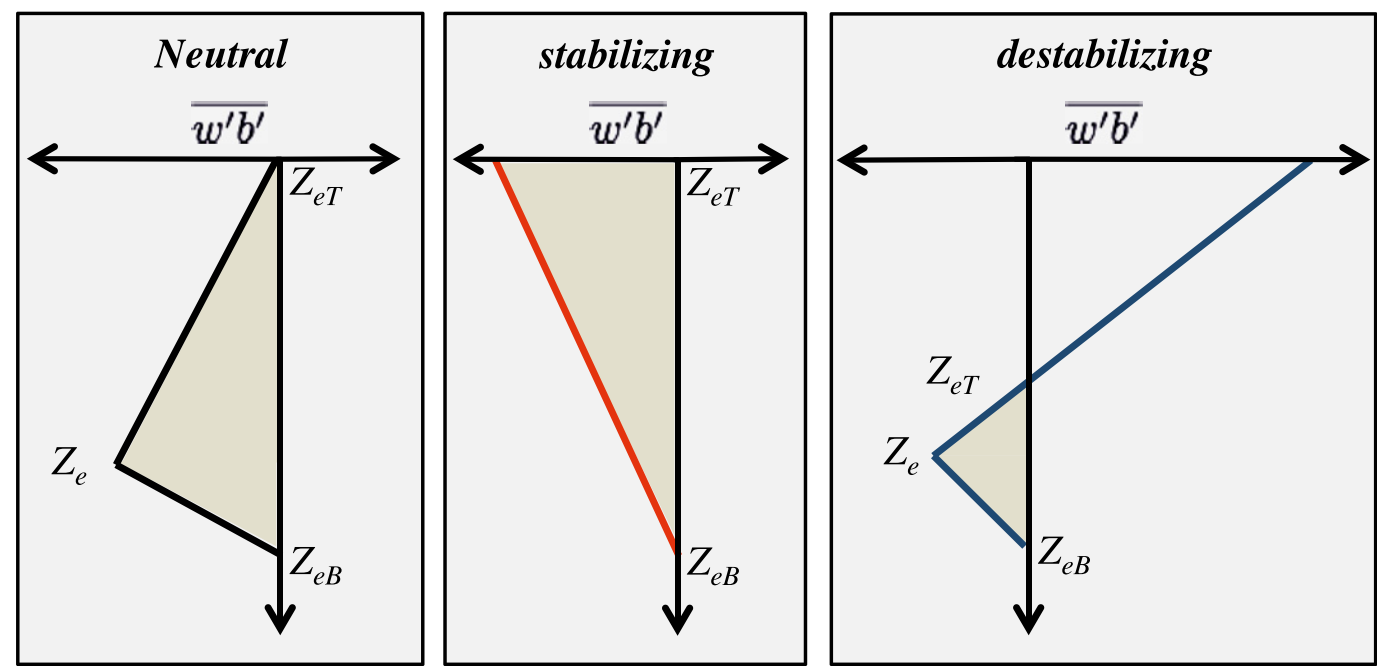

FIG. 1. A sketch of the approximate buoyancy flux $\overline{w^{\prime} b^{\prime}}$ profile observed during (left) neutral buoyancy, wind forced, (center) stabilizing buoyancy, wind forced, and (right) destabilizing buoyancy forced. The integral of the highlighted area is the quantity $M_{e}$ in this manuscript. The term $Z_{e T}$ is the depth of the top of the entrainment region, $Z_{e}$ is the depth of the peak (minimum) value of $\overline{w^{\prime} b^{\prime}}$, and $Z_{e B}$ is the depth of the bottom of the entrainment region. In stabilizing conditions the peak $\overline{w^{\prime} b^{\prime}}$ can be the surface flux and therefore there is no depth $Z_{e}$ given.

$$
\mathrm{Ri}_{b}(z)=\frac{\left[b(z)-b^{\mathrm{ref}}\right]|z|}{\left[u_{i}(z)-u_{i}^{\mathrm{ref}}\right]^{2}+v_{t}^{2}(z)},
$$

where the references values are based on a near-surface average and $v_{t}^{2}$ is the unresolved turbulence contribution to maintenance of the boundary layer depth. LF17 modify this calculation through a new parameterization of $v_{t}^{2}$, which results in a more consistent entrainment flux between KPP and their LES results (see their study and our section 4c). In ePBL the boundary layer thickness (and $K$ profile) are determined iteratively so that the integrated energetic effect of the vertical mixing is equivalent to the energy available $M_{e}$ [see Eq. (5) and RH18 for more detail]. The important difference between the KPP and ePBL approach is that $M_{e}$ is directly parameterized for ePBL, but is only indirectly related to the KPP bulk Richardson number method.

\section{3. $M_{e}$ and its parameterization}

We now discuss the parameterization of quantity $M_{e}$ as it pertains to ePBL and Eq. (5). It is important to clarify the physical significance of this quantity. To aid this discussion, simplified profiles of $\overline{w^{\prime} b^{\prime}}$ and the corresponding entrainment zone in destabilizing, neutral, and stabilizing regimes are illustrated in Fig. 1. The region enclosed by the negative part of the buoyancy flux profile constitutes the area that contributes to the integral $M_{e}$. In neutral and stabilizing regimes, $\overline{w^{\prime} b^{\prime}}$ is always less than or equal to zero, and therefore the quantity $M_{e}$ is equivalent to the total integral of $\overline{w^{\prime} b^{\prime}}$ within the boundary layer. In convective regions, $M_{e}$ isolates only the vertical fraction of the boundary layer where $\overline{w^{\prime} b^{\prime}}$ is negative and therefore contributes to net upward flux of less buoyant water.

The peak entrainment buoyancy flux is defined as the value of $\overline{w^{\prime} b^{\prime}}$ where it is most negative (represented as $\left.\overline{w^{\prime} b_{e}^{\prime}}\right)$. This value defines the entrainment depth $Z_{e}$, which is the depth where $\overline{w^{\prime} b^{\prime}}(z)=\overline{w^{\prime} b_{e}^{\prime}}$. In stabilizing conditions this definition of the entrainment depth and the entrainment buoyancy flux are not always applicable, as the surface buoyancy flux can be larger (more negative) than the buoyancy flux throughout the remainder of the entrainment zone. In these conditions, the boundary layer does not deepen, but recedes toward the Obukhov depth, $L_{O} \equiv-u_{*}^{3} / \kappa \overline{w^{\prime} b_{0}^{\prime}}$, where $\overline{w^{\prime} b_{0}^{\prime}}$ is the surface buoyancy flux, $\kappa$ is the von Kármán coefficient, and $u_{*}$ is the surface friction velocity related to the wind stress. By our definitions $M_{e}$ remains nonzero in such conditions due to the competition between mixing and the maintenance of the stable near-surface buoyancy gradient by the stabilizing surface flux. The bottom of the entrainment layer $Z_{e B}$ is equivalent to the base of the surface boundary layer $-H_{\mathrm{bl}}$, so these two parameters may be used interchangeably.

\section{a. $M_{e}$ in shear and convective turbulence}

RH18 presents a parameterization to predict $M_{e}$ from nondimensional relationships between 1) the surface fluxes, 2) characteristic time scales (the rotation and/or buoyancy frequency), and 3) a characteristic length scale $\left(H_{\mathrm{bl}}\right)$. The effects of these factors on $M_{e}$ is investigated 
by RH18 using a one-dimensional column model employing a form of the $k-\varepsilon$ turbulent mixing parameterization. The primary focus of their study was to develop a framework to parameterize OSBL turbulent mixing by predicting $M_{e}$ and subsequently using this quantity to constrain the turbulent mixing through the energetic effects on the mean state. This $M_{e}$ parameterization by RH18 applies for shear-driven stabilizing, sheardriven neutral, convective, and mixed shear/convective turbulence, but does not consider Langmuir turbulence. We therefore will first compare the $k-\varepsilon$ derived RH18 shear and convective turbulence parameterizations against the collection of non-Langmuir LES within this study (see section $2 \mathrm{a}$ for the LES description).

In $\mathrm{RH} 18, M_{e}$ is parameterized by dividing into mechanical processes and convective processes:

$$
-M_{e}=m_{*} u_{*}^{3}+n_{*} \int_{-H_{\mathrm{bl}}}^{0} \max \left(0, \overline{w^{\prime} b^{\prime}}\right) d z,
$$

where the first term represents a wind-driven shear turbulence contribution, and the second term represents the surface buoyancy loss contribution (the maximum function separating the convecting region from the entrainment zone). The shear turbulence proportionality coefficient $m_{*}$ is separated for stabilizing $(S)$ and nonstabilizing $(N)$ regimes as

$$
m_{*}=m_{* N} \Psi+m_{* S},
$$

where (as found in RH18)

$$
\begin{aligned}
m_{*_{N}} & =c_{N 1}\left\{1-\left[1+c_{N 2} \exp \left(-c_{N 3} H_{\mathrm{bl}}|f| / u_{*}\right)\right]^{-1}\right\}, \\
m_{* S} & =c_{S 1}\left[\min \left(0, \overline{w^{\prime} b_{0}^{\prime}}\right)^{2} H_{\mathrm{bl}} / u_{*}^{5}|f|\right]^{c_{S 2}}, \text { and } \\
\Psi & =1-c_{\Psi} \overline{\overline{w^{\prime} b_{0}^{\prime}}+2 m_{* N} u_{*}^{3} / H_{\mathrm{bl}}} .
\end{aligned}
$$

The empirical coefficients of RH18 are adopted in our experiments $\left(c_{N 1}=0.275, c_{N 2}=8, c_{N 3}=5, c_{S 1}=0.2\right.$, $c_{S 2}=0.4$, and $\left.c_{\Psi}=0.67\right)$. The proportionality $m_{*_{N}}$ is largest when the boundary layer depth is shallow relative to the Ekman depth, and reduces due to rotational effects as the boundary layer deepens. The presence of a stabilizing surface buoyancy flux increases $m_{*}$ (and therefore $M_{e}$ ) through $m_{* s}$, which represents mixing of near surface stable stratification. The presence of a destabilizing surface buoyancy flux has the opposite effect of a stabilizing flux, since it results in $\overline{w^{\prime} b^{\prime}}$ releasing $\mathrm{PE}$ via convection (in turn, no addition to $M_{e}$ occurs in this region). This reduction is represented by decreasing $m_{*}$ through multiplication by $0 \leq \Psi \leq 1$.
The shear-turbulence quantity represented by $m_{*}$ used here only represents the mean wind-driven boundary layer turbulent mixing and deliberately neglects transient variability in the mixing related to inertial oscillations. It therefore does not include representation of any effects of internal shear-turbulence generation mechanisms such as the inertial oscillations, equatorial undercurrents, and density overflow currents. As discussed in RH18, these processes and their contribution to mixing may be included via additional parameterizations, such as through employing the resolved-shear mixing parameterization of Jackson et al. (2008). For computing the mean shear-driven contribution to mixing from the LES results we only show the average over an inertial cycle, which helps to filter the impact of inertial oscillations on the turbulence.

\section{1) ESTIMATING $n_{*}$}

The value $n_{*}$ is found by RH18 to be between 0.04 and 0.08 based on both the $k-\varepsilon$ formulation and historical observations (e.g., Caughey and Palmer 1979, and studies cited therein). The LES experiments do not include any pure convection cases to provide a direct LES estimate of $n_{*}$ to compare with RH18, so we instead extrapolate to estimate this value. The value $n_{*}$ in a pure convective simulation can be found from Eq. (13):

$$
n_{*}=\frac{-M_{e}}{\int_{-H_{\mathrm{b} l}}^{0} \max \left(0, \overline{w^{\prime} b^{\prime}}\right) d z}
$$

To extrapolate LES to the convective limit we first define the total efficiency of the turbulence production to mixing $\left(M_{e}\right)$ as $e_{*}$, which includes the convection plus mechanical (shear) production terms:

$$
e_{*}=\frac{-M_{e}}{\int_{-H_{\mathrm{b} l}}^{0} \max \left(0, \overline{w^{\prime} b^{\prime}}\right) d z+\int_{-H_{\mathrm{b} 1}}^{0} \overline{w^{\prime} u_{i}^{\prime}} \partial_{z} \bar{u}_{i} d z}
$$

When the mechanical contribution tends to zero, $e_{*}$ is equivalent to $n_{*}$. We also define the buoyancy production fraction as the ratio of buoyancy TKE production to total TKE production:

$$
B_{\mathrm{Frac}}=\frac{\int_{-H_{\mathrm{bl}}}^{0} \max \left(0, \overline{w^{\prime} b^{\prime}}\right) d z}{\int_{-H_{\mathrm{bl}}}^{0} \max \left(0, \overline{w^{\prime} b^{\prime}}\right) d z+\int_{-H_{\mathrm{bl}}}^{0} \overline{w^{\prime} u_{i}^{\prime}} \partial_{z} \bar{u}_{i} d z}
$$

The buoyancy production fraction $B_{\text {Frac }}$ is zero during purely mechanical driven turbulence and tends to one for purely convective driven turbulence. Extrapolating 
the value of $e_{*}$ as $B_{\text {Frac }} \rightarrow 1$ can therefore provide a method to estimate $n_{*}$ from the LES.

A linear extrapolation of this ratio provides an estimate as the upper bound (blue dashed line, Fig. 2) and yields $n_{*}=0.06$ at $B_{\text {Frac }}=1$. However, the ratio may level off at $n_{*}=0.04$ (as indicated by two experiments that depart from this linear trend for $B_{\text {Frac }}>0.75$ ). Without additional experiments it is difficult to diagnose whether the departure from the trend is real or an artifact. Since the focus of this study is not convective turbulence, we will not investigate this value in detail, but instead test both values of $n_{*}^{a}=0.04$ and $n_{*}^{b}=0.06$ using all LES experiments.

\section{2) Estimating $m_{*}$ AND ITS COMPONENTS}

For the cases with a destabilizing surface buoyancy flux, $M_{e}$ can then be used to estimate $m_{*}$ from the LES via [combining Eqs. (13) and (14) and setting $m_{* S}=0$ ]:

$$
m_{*}=\frac{-M_{e}-n_{*} \int_{-H_{\mathrm{b} 1}}^{0} \max \left(0, \overline{w^{\prime} b^{\prime}}\right) d z}{\Psi u_{*}^{3}} .
$$

We show the result with and without inclusion of fraction $\Psi$ for both values of $n_{*}$ in Fig. 3. The cases without $\Psi$ (Figs. 3a,b) underestimate the curve fit for $m_{*}$ of RH18 by nearly a factor of 2 [dashed black line, Eq. (15a)]. Including the factor $\Psi$ significantly improves agreement with the RH18 curve fit, particularly at the value of $n_{*}=0.06$ more consistent with RH18 (Fig. 3d). The estimated value of $m_{*}$ from the LES at relatively larger values of $B_{\text {Frac }}$ where convection is more important and hence likely dominates uncertainty significantly exceeds the RH18 $m_{*}$ curve fit, but the cases with lowest $B_{\text {Frac }}$ fit well with the curve.

To evaluate the RH18 parameterization against all the cases from LF17 with no Langmuir turbulence we compare $M_{e}$ from RH18 to that diagnosed from LES in Fig. 4a. The results predicted using the RH18 formulation are consistent with the results computed from the LES over the entire range of convective, stabilizing, and shear turbulence regimes. We therefore conclude that the RH18 parameterization for $m_{*}$ (including components $m_{*_{N}}, m_{* S}$, and $\Psi$ ) and $n_{*}=0.06$ accurately predict $M_{e}$ compared to the LES in non-Langmuir conditions, even for the cases where $m_{*}$ was overestimated in Fig. 3d.

In Fig. 4 we also compare the RH18 parameterization against the full set of simulations from LF17 including Langmuir turbulence (Fig. 4b). We find that a significant fraction of $M_{e}$ is underpredicted by RH18 in the simulations with Langmuir turbulence, particularly those in strongly mechanically forced conditions (darker

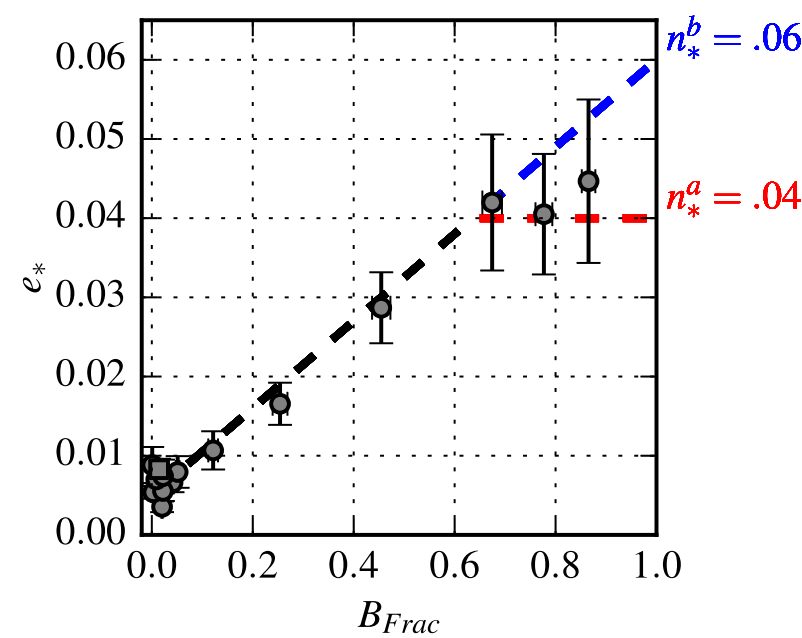

FIG. 2. The ratio of $-M_{e}$ to total TKE production [ $e_{*}$, Eq. (17)], vs the buoyancy production fraction $\left[B_{\text {Frac }}\right.$, Eq. (18)]. The blue dashed line assumes that this fraction linearly approaches $n_{*}=0.06$ in purely convective turbulence, while the red dashed line assumes this fraction levels off at $n_{*}=0.04$

markers). The RH18 parameterization is inadequate to capture the effect of Langmuir turbulence without modification. A proposed modification is presented in the following section.

\section{b. Enhancement to $M_{e}$ in Langmuir turbulence}

We introduce a new parameter $m_{*_{\mathrm{LT}}}$ to extend Eq. (13) to include the effects of Langmuir turbulence by writing $M_{e}$ in the modified form:

$-M_{e}=\left(m_{*}+m_{* \mathrm{LT}}\right) u_{*}^{3}+n_{*} \int_{-H_{\mathrm{bl}}}^{0} \max \left(0, \overline{w^{\prime} b^{\prime}}\right) d z$.

Scaling arguments suggest that Stokes production of TKE should increase approximately as $u_{*}^{2}\left|\tilde{u}_{i 0}\right|$ (Belcher et al. 2012), suggesting $m_{*_{\text {LT }}}$ should be formulated to capture this enhancement (scaling with $\mathrm{La}_{t}^{-2}$ ). However, the quantity $m_{*_{\mathrm{LT}}}$ is not strictly representing only the addition of mixing due to Langmuir turbulence production, since the Eulerian current shear and therefore $m_{*}$ are reduced due to the presence of Stokes shear (e.g., McWilliams et al. 1997; Li and Fox-Kemper 2017). Therefore, $m_{* \mathrm{LT}}$ represents the net enhancement of $M_{e}$ beyond the level that would occur in shear-only turbulence without considering the fractional contribution of Langmuir turbulence and shear turbulence.

The value of $m *_{\text {LT }}$ will vary as a function of the Langmuir number. The form of the Langmuir number appropriate for parameterizing $m_{*_{\mathrm{LT}}}$ is also investigated here. In aligned wind and wave conditions, previously published turbulence scalings primarily scale properties using $\mathrm{La}_{t}$ as defined in Eq. (1) or $\mathrm{La}_{\mathrm{SL}}$ as defined in 


\section{$B_{\text {Frac }}$}

0.00

0.01

0.02

0.03

0.04

0.05
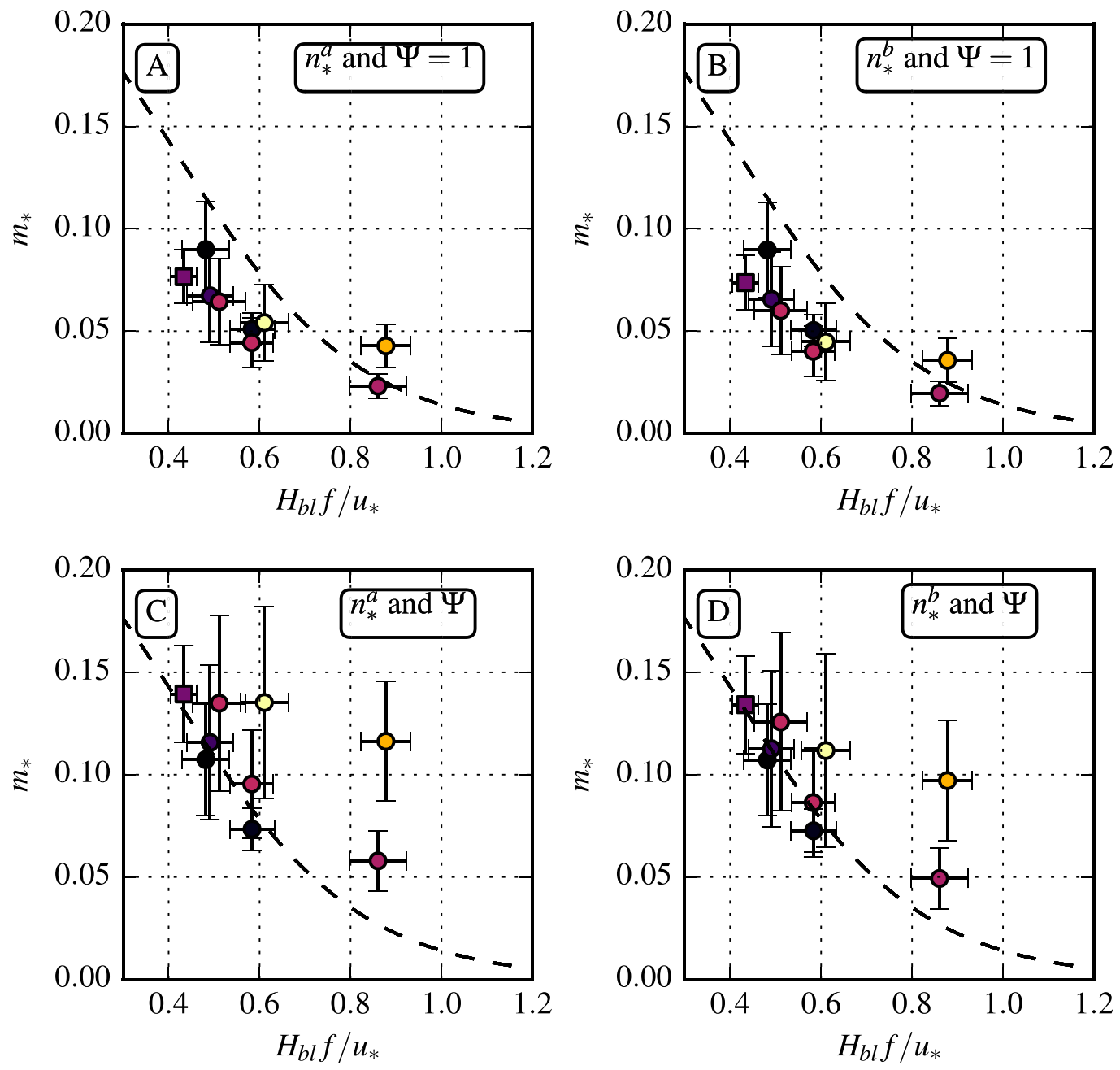

FIG. 3. The derived value of $m_{*}$ from LES vs the ratio of the OSBL thickness to the Ekman length scale. The dashed line is the empirical fit of RH18 $\left[m_{* N}\right.$, Eq. (15a)]. Shown are (a) $n_{*}=0.04$ without $\Psi$, (b) $n_{*}=0.06$ without $\Psi$, (c) $n_{*}=0.04$ with $\Psi$, and (d) $n_{*}=0.06$ with $\Psi$, where the correction $\Psi$ [Eq. (15c)] parameterizes the effect of convective turbulence on shear turbulence. In this and subsequent figures, the marker shading represents the buoyancy production fraction [(Eq. (18)], except where otherwise indicated.

Eq. (2). In general, $\mathrm{La}_{\mathrm{SL}}$ has been the preferred parameter for scaling integrated turbulence metrics in previous studies (Harcourt and D'Asaro 2008; Van Roekel et al. 2012; Reichl et al. 2016; Li and FoxKemper 2017). However, different metrics can scale better with different forms and exponential relations of the Langmuir number (e.g., Reichl et al. 2016), while only the scaling of Stokes production is bounded by theory to scale as $u_{*}^{3} \mathrm{La}_{t}^{-2}$ (e.g., Grant and Belcher 2009).

To diagnose $m_{* \text { LT }}$ we can rearrange Eq. (20):

$$
m_{*_{\mathrm{LT}}} u_{*}^{3}=-M_{e}-m_{*} u_{*}^{3}-n_{*} \int_{-H_{\mathrm{bl}}}^{0} \max \left(0, \overline{w^{\prime} b^{\prime}}\right) d z
$$

Here we investigate different functional forms of the Langmuir number to best predict $m_{* \mathrm{LT}}$ and show four of them in Fig. 5. The product $m_{*_{\mathrm{LT}}} u_{*}^{3}$ is diagnosed from Eq. (21) for all LES cases and plotted against $\mathrm{La}_{\mathrm{SL}}^{-p}$, where $p$ is an empirically found exponent. The value $p=$ 2 (Fig. 5a) is equivalent to the scaling of Stokes production (e.g., Belcher et al. 2012): 


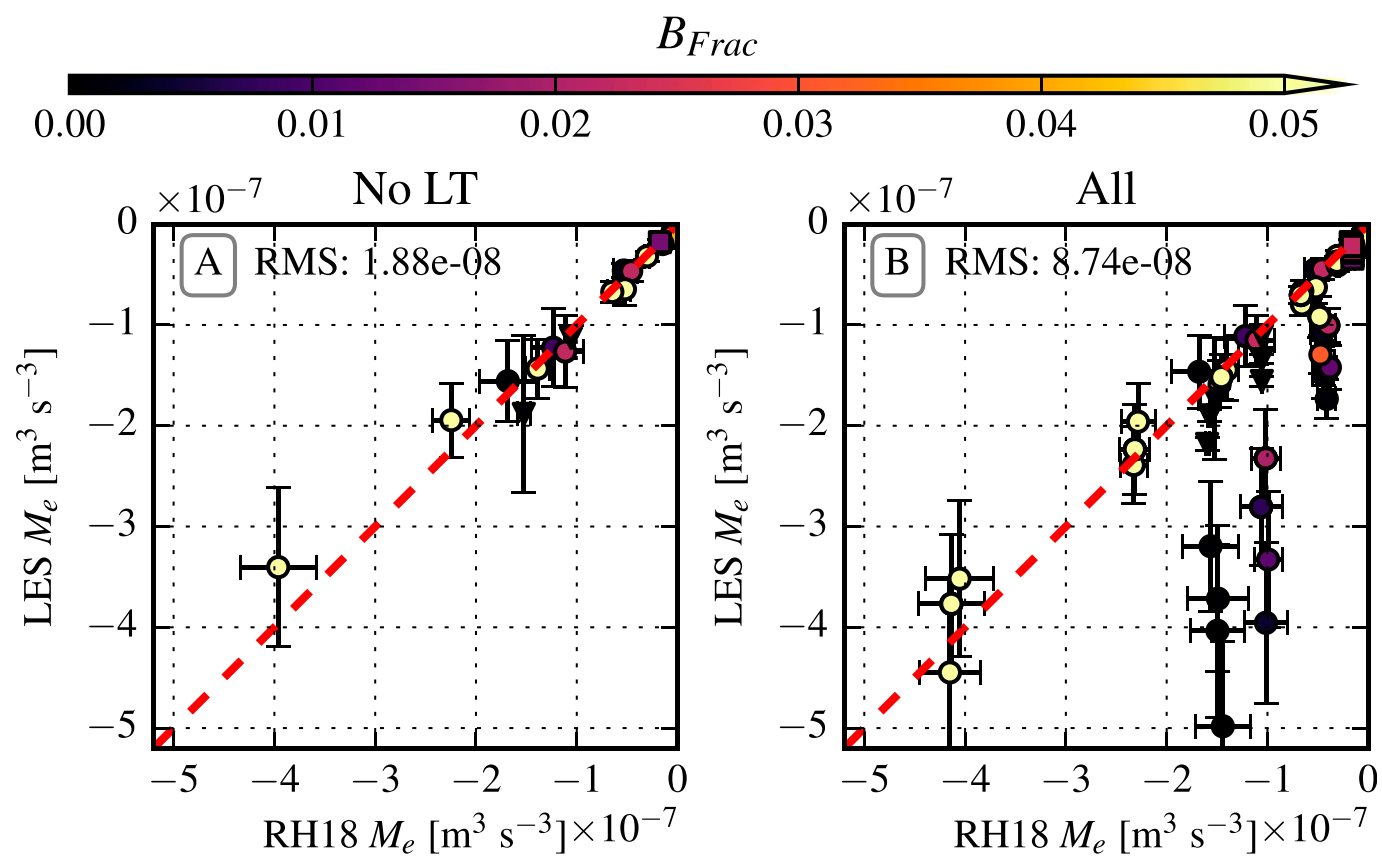

FIG. 4. (a) $M_{e}$ computed from the non-Langmuir LES vs $M_{e}$ predicted from RH18. (b) As in (a), but also including the Langmuir LES cases. The dashed red line represents a 1:1 fit.

$$
f_{1}\left(\mathrm{La}_{x}\right)=\mathrm{La}_{\mathrm{SL}}^{-2}
$$

Using $p=1$ (Fig. 5b), that is,

$$
f_{2}\left(\mathrm{La}_{x}\right)=\mathrm{La}_{\mathrm{SL}}^{-1}
$$

appears to yield an improved linear fit. However, significant scatter remains that correlates with $B_{\text {Frac }}$, indicated by the shading. To reduce this scatter we tested additional parameter dependency using a matrix of nondimensional parameter combinations from $u_{*}, B_{f},|f|$, and $H_{\mathrm{bl}}$, yielding most significant reduction in RMS difference from LES with the ratio $\left|B_{f}\right| / u_{*}^{2}|f|$. A physical interpretation for this nondimensional relationship comes from scaling arguments of buoyancy and shear driven production, which roughly scale as $B_{f}$ and $u_{*}^{3} / H_{\mathrm{bl}}$, respectively. The limit where $H_{\mathrm{bl}}$ approaches the Ekman length, $L_{E} \equiv u_{*} /|f|$ (setting the upper bound for shear production in rotating, shearonly turbulence), yields a ratio for scaling the buoyancy to shear driven production ratio of $B_{f} / u_{*}^{2}|f|$. The absolute value of $B_{f}$ is introduced as this scaling works to capture variability of $M_{e}$ in both stabilizing and destabilizing $B_{f}$, though limited stabilizing cases are tested here. This ratio will cause the effect of Langmuir turbulence to be reduced (increasing the effective $\left.\mathrm{La}_{\mathrm{SL}}\right)$ as $\left|B_{f}\right|$ increases or $u_{*}$ or $|f|$ decreases, though future investigation to refine behavior as $|f| \rightarrow$ 0 is needed. We express this ratio equivalently as the ratio of the Ekman depth and the absolute Obukhov depth, $\left|L_{O}\right|=u_{*}^{3} / \kappa\left|B_{f}\right|$ and therefore propose a form

$$
f_{3}\left(\operatorname{La}_{x}\right)=\left[\operatorname{La}_{\mathrm{SL}}\left(1+C_{1} \frac{L_{E}}{\left|L_{O}\right|}\right)\right]^{-1},
$$

which produces a compact linear trend with the diagnosed $m_{*_{\mathrm{LT}}} u_{*}^{3}$ (Fig. 5c). We repeat the procedure with $\mathrm{La}_{t}$ and find

$$
f_{4}\left(\operatorname{La}_{x}\right)=\left[\operatorname{La}_{t}\left(1+C_{2} \frac{L_{E}}{\left|L_{O}\right|}\right)\right]^{-3 / 2},
$$

which is shown in Fig. 5d.

In each case an empirical coefficient is fit based on the linear fit to the data, such that

$$
m_{*_{\mathrm{LT}}} u_{*}^{3}=c_{\mathrm{LT}} f_{x}\left(\mathrm{La}_{x}\right) u_{*}^{3},
$$

where the coefficient $c_{\mathrm{LT}}$ is the slope in each respective panel of Fig. 5. This fit is intentionally conducted for the dimensional data to bias toward the stronger mechanical mixing events when the Langmuir turbulence signal is less susceptible to noise. To confirm the validity of the results across the parameter space, fits for the same relations is shown to nondimensional parameters in Fig. 6.

In Fig. 7, $M_{e}$ estimated from Eq. (20) with $m_{* \text { LT }}$ being a function of either $\mathrm{La}_{t}$ as in Eq. (25) or $\mathrm{La}_{\mathrm{SL}}$ as in Eq. (24) are compared with $M_{e}$ diagnosed from LES. 


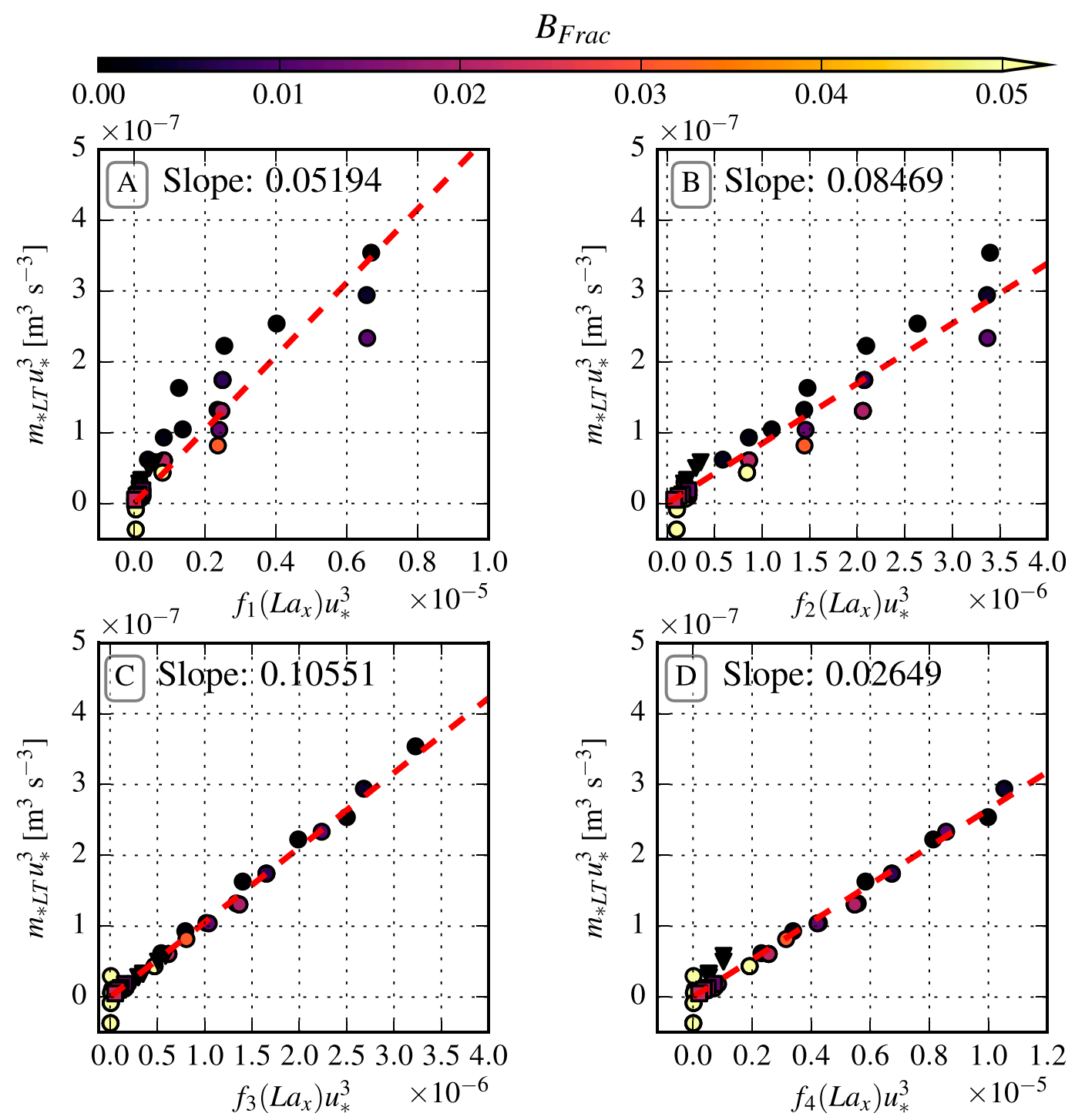

FIG. 5. Shown is $m_{*_{\mathrm{LT}}} u_{*}^{3}$ vs various functions related to the Langmuir number. See (a) Eq. (22) for $f_{1}$, (b) Eq. (23) for $f_{2}$, (c) Eq. (24) for $f_{3}$, and (d) Eq. (25) for $f_{4}$. The dashed red line represents a linear fit with the slope given in the upper left of each panel and the intercept set to zero. The slopes have each been confirmed statistically significant beyond $95 \%$ confidence.

Both appear to reproduce $M_{e}$ in the LES, though $\mathrm{La}_{\mathrm{SL}}$ is preferred as it yields smaller RMS error. This is consistent with $\mathrm{La}_{\mathrm{SL}}$ being preferred in previous studies based on scaling of enhanced $\overline{w^{\prime} w^{\prime}}$ (see Harcourt and D'Asaro 2008; Van Roekel et al. 2012; Reichl et al. 2016), $K$ (Reichl et al. 2016), and $\overline{w^{\prime} b_{e}^{\prime}}$ (see $\mathrm{Li}$ and FoxKemper 2017).

\section{Results}

In this section we will compare the detailed results of simulations with ePBL including the modification proposed in the previous section against a subset of the LES simulation. We will also include the LF17 model in this comparison and compare and contrast the two approaches in detail.

\section{a. Impact in one-dimensional simulations}

Here we investigate the ability of ePBL with the new $m_{* \text { LT }}$ parameterization to reproduce the enhanced mixing due to Langmuir turbulence. We do not investigate the role of Langmuir turbulence to enhance the local value of $K$ throughout the water column in this study (see Reichl et al. 2016). We therefore introduce two versions of ePBL that are tested in detail in this section. The first version (ePBL-LT) uses ePBL 


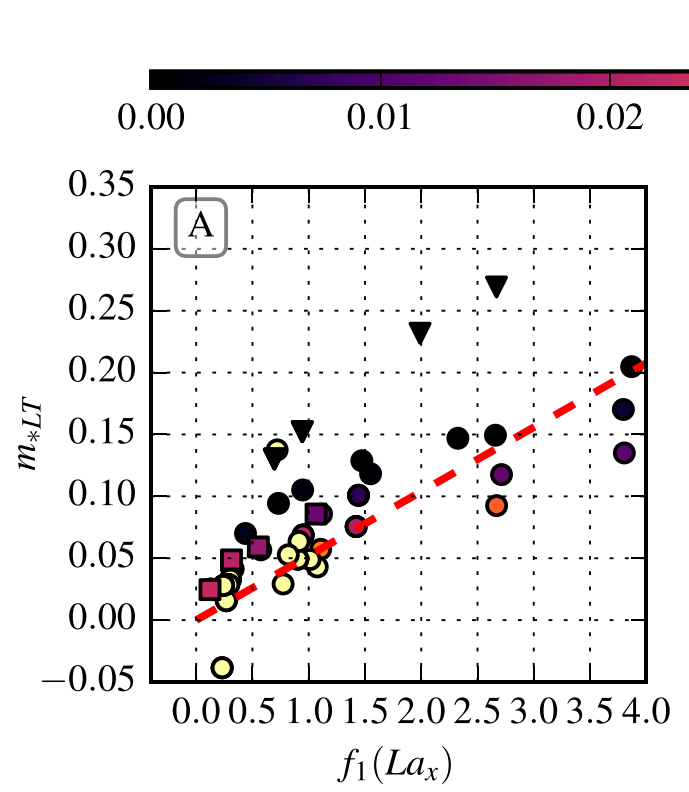

B Frac
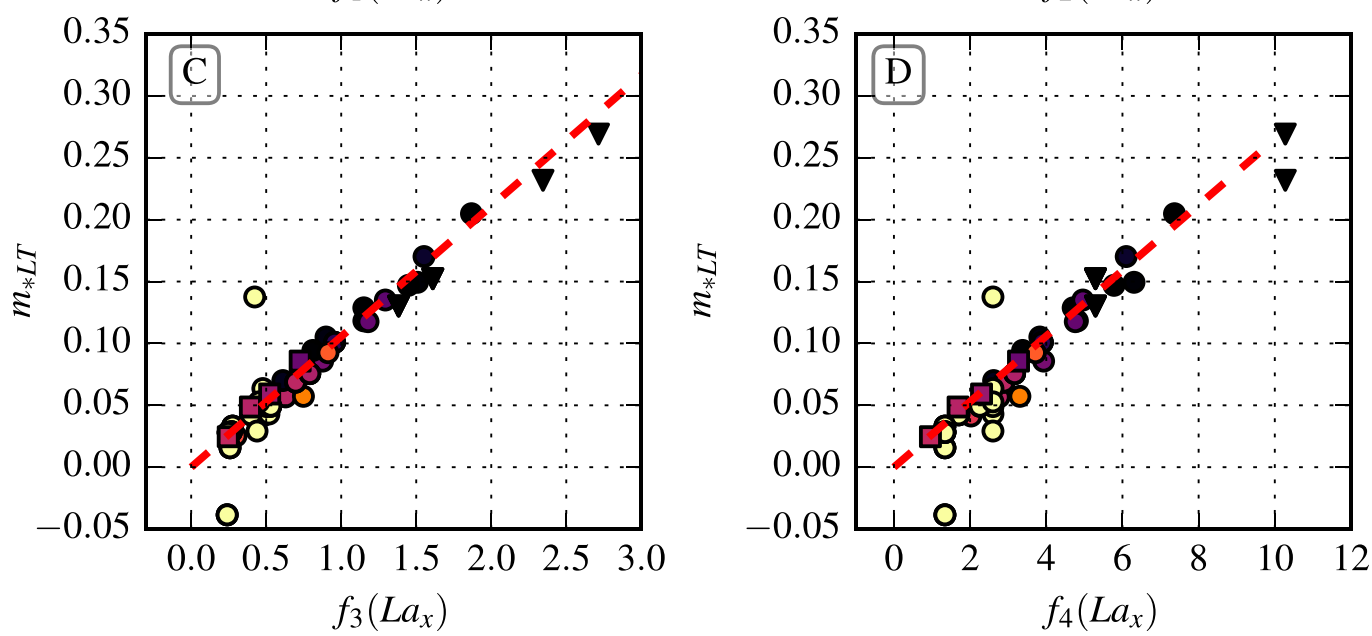

FIG. 6. As in Fig. 5, but here the data are nondimensionalized by $u_{*}^{3}$. Note that buoyancy driven cases are cut off by the $y$-axis lower limit. In these cases $u_{*}$ is small relative to the buoyancy forcing so the disagreement is amplified by the normalization (and error is likely related to variability in $n_{*}$ ).

as described in section $2 b$. The second version (ePBLLT-Bulk) is similar to the first, but use arbitrarily large coefficients in Eqs. (8) and (9), thereby mimicking a well-mixed boundary layer parameterization [arbitrarily large $K$ by Eq. (6)].

These two approaches bound the possible modifications to $K$, since the first method will underestimate $K$ in the presence of Langmuir turbulence, while the second method is the maximum possible $K$ and therefore an overestimation. For comparison with the LF17 study we also employ the CVMix-KPP model using the LF17 modification for computing the boundary layer depth. We investigate results from experiments with $10 \mathrm{~m} \mathrm{~s}^{-1}$ wind speed in these results, since the net Langmuir turbulence effect tends to be most profound due to the strong wind and wave forcing (experiments 5, 9, and 12).

In Fig. 8, the temperature profile from two experiments with small (experiment $5,-5 \mathrm{~W} \mathrm{~m}^{-2}$, left panels) and large (experiment $12,-50 \mathrm{~W} \mathrm{~m}^{-2}$, right panels) convective contributions are shown. In each case the results for no wave (solid) and wave age $=1.2$ (dashed) are plotted on the same axis at the similar time from each LES and the one-dimensional simulation. The length of the LES simulations in all cases are not the same, so therefore we present all results at the conclusion of the shortest duration LES run (1.07 simulation days). The LES and one-dimensional model turbulent spinup times are not comparable, meaning it may not be valid to compare the simulations 


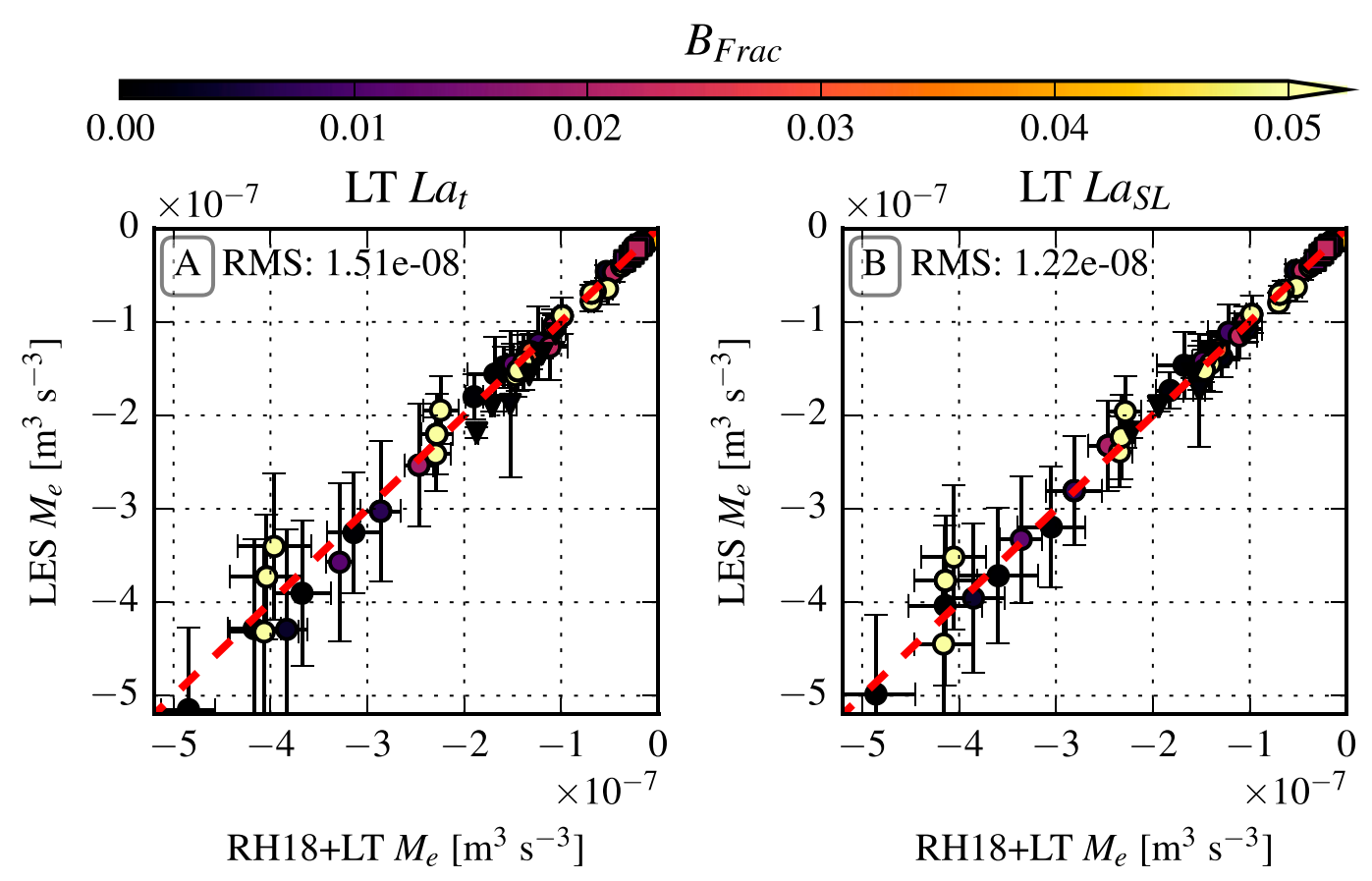

FIG. 7. $M_{e}$ computed from the LES results vs $M_{e}$ predicted using (a) the turbulent Langmuir number formulation [Eq. (25)] and (b) the surface-layer averaged Langmuir number formulation [Eq. (24)]. The red dashed line represents the 1:1 fit, where the estimate and the derived value are the same.

at the same time step. However, we also investigated plotting LES/MOM6 results at the time of equivalent $\mathrm{PE}$ change (which is exactly comparable due to their identical vertical domain configuration) and found virtually identical results.

In general, the temperature profile comparisons suggest a few key conclusions. First, both the KPP and ePBL parameterizations are able to reasonably predict the entrainment without Langmuir turbulence in both experiments shown here (this is compared directly in Fig. 9). Second, the ePBL approach captures the enhanced cooling and entrainment due to Langmuir turbulence reasonably well for both simulations, while the KPP approach underestimates the total excess cooling in the Langmuir turbulence case. Third, the vertical structure of the mixing coefficient has a significant impact on the ability of the parameterization to predict a similar temperature profile at a similar time, indicating disagreement between the locality of where the mixing occurs in each experiment (e.g., note the different locations of relative vertical temperature homogenization between the green and red lines in both experiments). Finally, the excess homogenization of the temperature profile near the surface by Langmuir turbulence can only be captured by explicitly increasing $K$ near the surface, a point particularly emphasized by the poor performance of the red-dashed profile compared to the solid red profile in Figs. $8 \mathrm{c}$ and $8 \mathrm{~d}$. We note that increasing $K$ following Reichl et al. (2016) in ePBLLT can improve this bias, but since their study only investigated strongly shear-driven mixing under hurricanes we do not present this result in detail but propose it as a motivation for future research.

There are two significant differences between the KPP method and the ePBL method that contribute to the differences seen in this set of one-dimensional simulations. First is that KPP also includes nongradient turbulent buoyancy flux (see, e.g., Van Roekel et al. 2018). The impact of the nongradient mixing is seen most clearly in the difference between the ePBL (red) and KPP (green) temperature profile in the stronger convection case (Figs. 9b,d). Second is that ePBL is numerically more robust to model time step and vertical resolution and better able to capture the enhancement of mixing due to Langmuir turbulence in these simulations where vertical resolution is much higher than typical applications in climate models. The second difference is entirely due to the sensitivity of KPP to implementation details, specifically the sensitivity of $v_{t}^{2}$ in the bulk Richardson number [Eq. (11)] to grid discretization (Van Roekel et al. 2018), which is amplified by including the $\overline{w^{\prime} b_{e}^{\prime}}$ scaling in LF17 to account for the effect of Langmuir turbulence. The $\overline{w^{\prime} b_{e}^{\prime}}$ relationships found in LF17 were derived from the same LES results considered here and therefore are consistent with the $M_{e}$ relationships found here (this is 

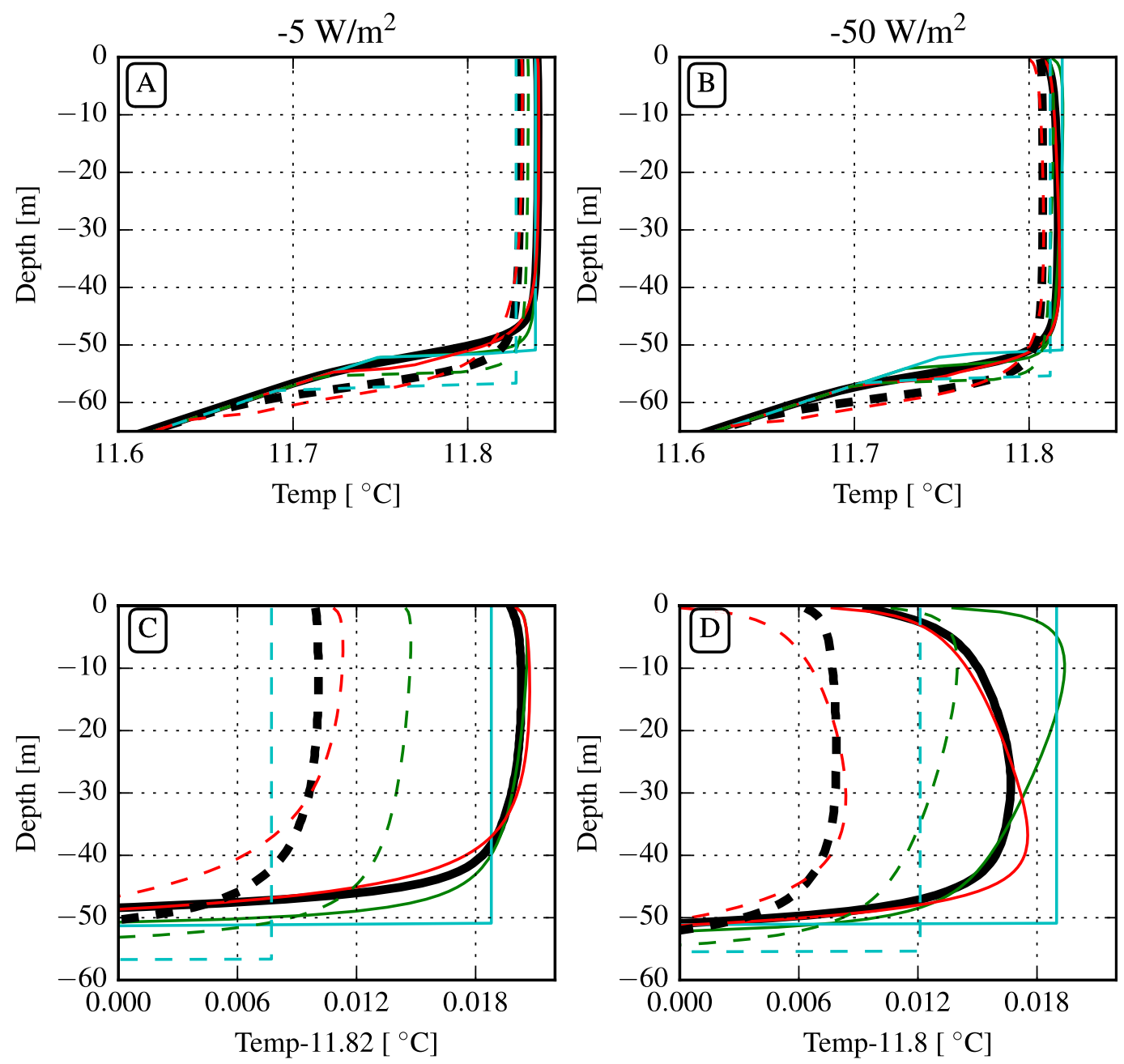

FIG. 8. Comparison of LES (black) and MOM6 for (a),(c) Experiment 5 and (b),(d) Experiment 12 with KPP-LF17 (green), ePBL (red), and ePBL-Bulk (cyan) (see also the legend in Fig. 9 for line-color meanings) for no wave (solid) and strong wave (dashed) cases. Panels (a) and (c) and panels (b) and (d) show identical data, but with the axis changed to emphasize different portions of the water column.

explored in detail in section $4 \mathrm{~b}$ ). The disagreement between the simulations with ePBL and KPP here only emerge when the LF17 $\overline{w^{\prime} b_{e}^{\prime}}$ parameterization is applied in KPP, and may appear bigger or smaller depending on the choice of grid discretization (e.g., Van Roekel et al. 2018). This difference demonstrates a strength of ePBL versus KPP for reducing model grid sensitivity by reducing dependence on quantities discretized on the calling model's vertical grid.

We next investigate bulk properties of the model simulation including the predicted rate of integrated potential energy change and the rate of temperature cooling at the surface. These two metrics indicate different aspects of the performance of the vertical mixing parameterizations, which when taken together indicate the ability of a parameterization to accurately simulate the entire ocean surface boundary layer. To mitigate the difference between the LES and one-dimensional model spinup process of turbulence here, we focus on these specific results averaged over the time period of the final inertial period in each LES model. The LES profiles are not held constant during the turbulence spinup, which would complicate comparison of the absolute change of surface temperature and potential energy relative to the initial condition. Therefore, we focus our investigation on the mean of the instantaneous rate of change of these quantities for a time period not including the spinup processes. We show the results from cases without waves (with squares, triangles, and diamonds indicating the three different experiments) and with the strongest wave forcing (wave age $=1.2$, circles with like experiments connected with lines for clarity). We first show the 

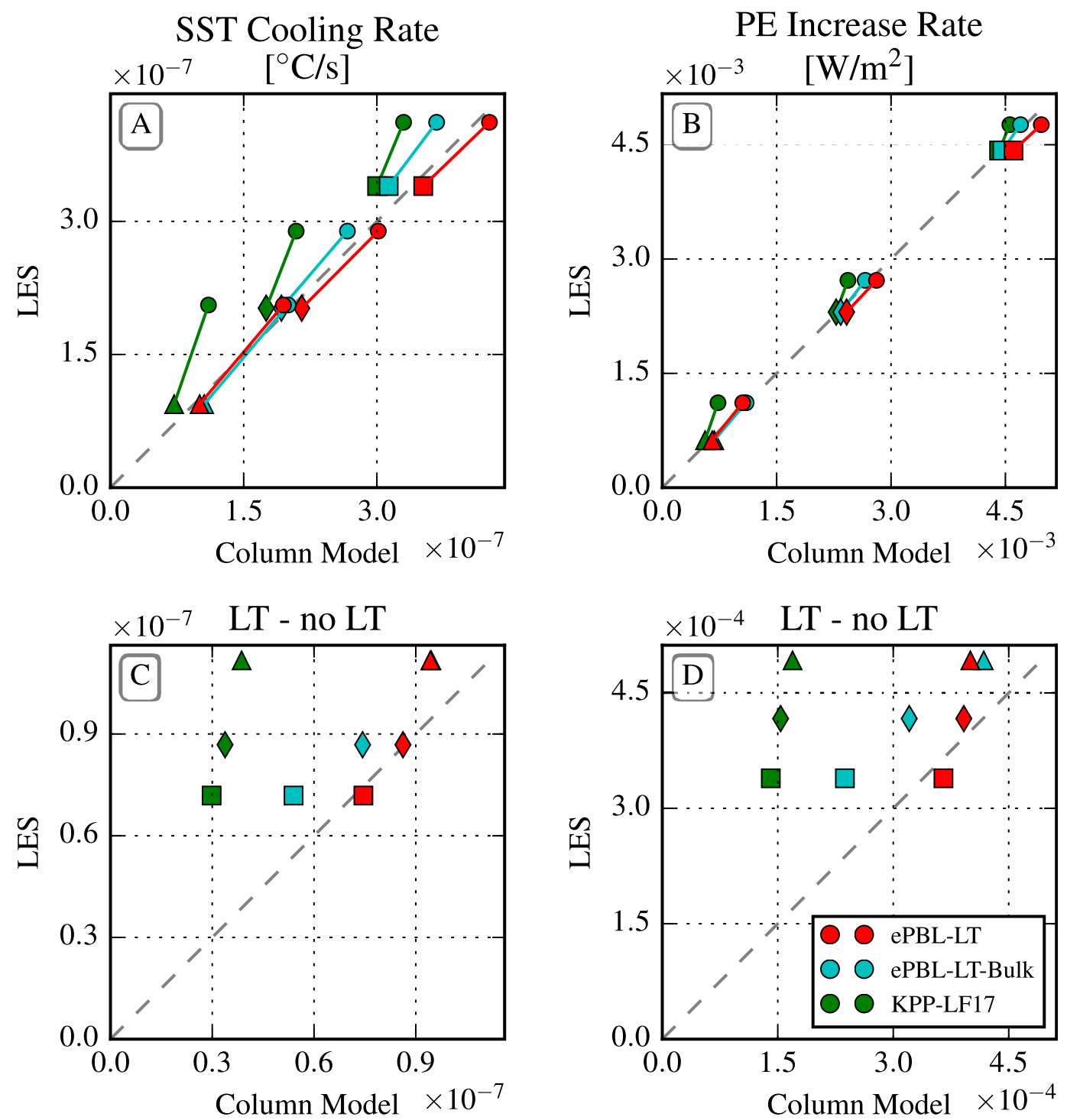

FIG. 9. Comparison of one-dimensional MOM6 simulations with ePBL-LT, ePBL-LT-Bulk, and KPP-LF17 to LES for Experiments 5, 9, and 12 without Stokes drift (triangles: Exp. 5, diamonds: Exp. 9, and squares: Exp. 12) and with Stokes drift using wave-age $=1.2$ (circles with lines connecting to corresponding simulations without Stokes drift). (a) The time rate of change of SST and (b) the time rate of change of PE. (c),(d) As in (a) and (b), but subtracting the no Stokes drift case in each simulation from the case with Stokes drift, thus showing the difference due to including Langmuir turbulence.

surface temperature rate of change in the top grid cell (SST, with identical top gridcell thickness between LES and one-dimensional model), where we use identical vertical discretization in the one-dimensional model and the LES (Fig. 9a) for equivalence. We see that the ePBL-LT-Bulk and ePBL-LT simulations perform best for predicting consistent SST tendency both with and without Langmuir turbulence (cyan and red markers), with the LES result bounded between the ePBL-LT and ePBL-LT-Bulk result. These results indicate that further improved SST cooling rate simulation could be achieved through combining the ePBL-LT approach with a localized (in the vertical) mixing enhancement. The PE increase rate plots (Fig. 9b) show that all three experiments perform roughly equally well, with the ePBL-LT-Bulk simulations being between the KPP and ePBL-LT result.

To more carefully examine the effect of Langmuir turbulence in the models we then take the difference between the results with and without Stokes drift (Figs. 9c,d). In this case we find that ePBL-LT performs better for predicting the change in both SST-cooling 
and PE increase rate due to Langmuir turbulence. The offset between the LES and column model result grows for both KPP and ePBL-LT as the contribution due to Langmuir turbulence grows (Figs. 9c,d). However, the ePBL-LT-Bulk does not show a similar increase in error, again suggesting that the localized mixing enhancement can further improve these results.

\section{b. The relationship between $M_{e}$ and $\overline{w^{\prime} b_{e}^{\prime}}$}

To modify KPP, LF17 formulated a parameterization based on optimized prediction of $\overline{w^{\prime} b_{e}^{\prime}}$ relative to LES:

$$
-\overline{w^{\prime} b_{e}^{\prime}}=0.17 \frac{u_{*}^{3}}{H_{\mathrm{bl}}}\left(1+0.49 \mathrm{La}_{\mathrm{SL}}^{-2}\right)+0.15 \overline{w^{\prime} b_{0}^{\prime}} .
$$

This relation was used by LF17 to modify the unresolved turbulent velocity shear in the KPP bulk Richardson number in Eq. (12) to better represent turbulent entrainment in the presence of both convective and Langmuir turbulence. In the ePBL approach of this study we investigate the quantity $M_{e}$, the integral of the buoyancy flux over the entrainment region (see Fig. 1). To compare our results with LF17, we investigate the relationship between $M_{e}$ and $\overline{w^{\prime} b_{e}^{\prime}}$ in this section.

We first plot $M_{e}$ [Eq. (3)] against $\overline{w^{\prime} b_{e}^{\prime}}$ as diagnosed from the LES in Fig. 10a, which shows that the relationship between the two quantities is not linear. To better understand this nonlinear relationship, we investigate details of the buoyancy flux profile demonstrated by Fig. 1. The depth below where the buoyancy flux first becomes negative is the top of the entrainment layer $Z_{e T}$, which can be diagnosed within the LES result. The region between $Z_{e T}$ and $Z_{e}$ makes up the upper region of the entrainment layer. There exists a secondary region below $Z_{e}$, where the buoyancy flux remains negative (indicating active work against gravity) but increases (becomes less negative) moving downward to the base of the entrainment layer $Z_{e B}$ where the buoyancy flux becomes zero. The secondary region contributes a smaller fraction of $M_{e}$ in the shear-driven case (Fig. 1, left panel), but can make up roughly half of $M_{e}$ in the convective case (Fig. 1, right panel). The buoyancy flux profile in the entrainment layer approximately forms a pair of triangular shaped regions, approximately equating the mean buoyancy flux within the entrainment layer with $\overline{w^{\prime} b_{e}^{\prime}} / 2$ (see also vanZanten et al. 1999). The $M_{e}$ is found from this mean buoyancy flux multiplied by the thickness of this layer $\left(H_{e}=Z_{e T}-Z_{e B}\right)$ :

$$
M_{e} \equiv \int_{z_{\mathrm{eB}}}^{Z_{\mathrm{eT}}} \overline{w^{\prime} b^{\prime}} d z=\left\langle\overline{w^{\prime} b^{\prime}}\right\rangle_{H_{e}} H_{e} \approx \frac{\overline{w^{\prime} b_{e}^{\prime}}}{2} H_{e} .
$$

A major source of variability in the relationship between $M_{e}$ and $\overline{w^{\prime} b^{\prime}}$ is therefore the difference in entrainment layer thickness $H_{e}$ between shear and convective regimes (as demonstrated in Fig. 1).

We can rewrite Eq. (28) by splitting the upper and lower region:

$$
M_{e} \approx \overbrace{\frac{w^{\prime} b_{e}^{\prime}}{2}\left(Z_{e T}-Z_{e}\right)}^{\text {Top region }}+\overbrace{\frac{w^{\prime} b_{e}^{\prime}}{2}\left(Z_{e}-Z_{e B}\right)}^{\text {Bottom region }} .
$$

Following RH18, the thickness of the entrainment layer can be estimated through trigonometric arguments depending only on the surface buoyancy flux, entrainment buoyancy flux, and the entrainment depth. The top of the entrainment layer is approximated by

$$
Z_{e T} \approx\left(1-\frac{-\overline{w^{\prime} b_{e}^{\prime}}}{\overline{w^{\prime} b_{0}^{\prime}}-\overline{w^{\prime} b_{e}^{\prime}}}\right) Z_{e} .
$$

Using Eq. (30) in the first (top region) term in Eq. (29), we find

$$
\int_{Z_{e}}^{Z_{\mathrm{eT}}} \overline{w^{\prime} b^{\prime}} d z \approx \frac{\overline{w^{\prime} b_{e}^{\prime}}}{2} Z_{e}\left(-\frac{-\overline{w^{\prime} b_{e}^{\prime}}}{\overline{w^{\prime} b_{0}^{\prime}}-\overline{w^{\prime} b_{e}^{\prime}}}\right) .
$$

To close Eq. (29), we approximate the depth of the bottom of the entrainment region, $Z_{e B}$, from $Z_{e}$ and $\overline{w^{\prime} b_{e}^{\prime}}$. The buoyancy flux converges to zero at depth $Z_{e B}$ using our definition of the base of the OSBL (internal wave-breaking or interior-driven turbulence would complicate this scenario, but we neglect internal processes for this discussion). For simplicity, this depth is assumed to scale linearly by a coefficient $A$ with the entrainment depth:

$$
Z_{e B} \approx Z_{e}(1+A) .
$$

Substituting Eq. (32) for the second (bottom) term in Eq. (29) gives

$$
\int_{Z_{e B}}^{Z_{e}} \overline{w^{\prime} b^{\prime}} d z \approx \frac{\overline{w^{\prime} b_{e}^{\prime}}}{2}-Z_{e} A .
$$

Using Eqs. (31) and (33), we can estimate $A$ from the LES results by

$$
A=-\left[\frac{2 M_{e}}{\overline{w^{\prime} b_{e}^{\prime}} Z_{e}}-\left(-\frac{-\overline{w^{\prime} b_{e}^{\prime}}}{\overline{w^{\prime} b_{0}^{\prime}}-\overline{w^{\prime} b_{e}^{\prime}}}\right)\right] .
$$

We hypothesize that $A$ varies based on the buoyancy TKE production fraction $B_{\text {Frac }}$ [Eq. (18)]. Through a 

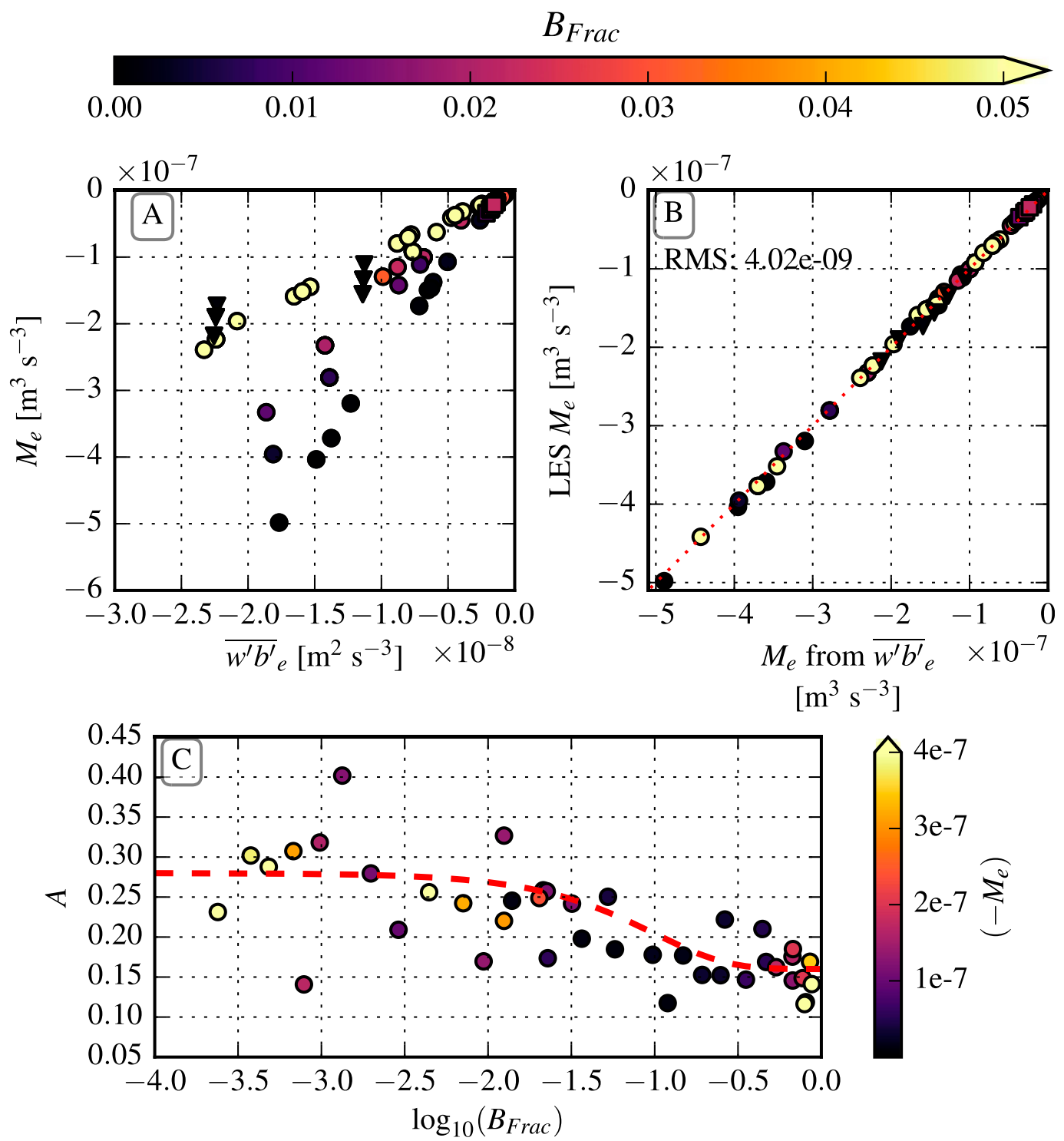

FIG. 10. (a) Integrated entrainment buoyancy flux $M_{e}$ vs the peak entrainment buoyancy flux $\overline{w^{\prime} b_{e}^{\prime}}$ from the LES cases of LF17 and the three additional simulations for this study. (c) Comparison of factor $A$ for the lower entrainment region vs $\log _{10}$ of the buoyancy production fraction. The red dashed line represents the empirical fit to the data based on minimizing RMS (thus biasing the line toward darker colored circles that represent more negative $M_{e}$ ). (b) The peak entrainment buoyancy flux from the LES vs that predicted using Eq. (36). In (a) and (b), the color represents the fraction of TKE production due to buoyancy [explained in detail in section 4b, Eq. (18)], while in (c) the color represents the total $M_{e}$. Data indicated with circles represent the LF17 data, squares represent the experiment with the reduced Coriolis parameter, and triangles represent the cases with surface heating.

series of least-mean square adjustments based on the total integrated mixing (the final version appearing in Fig. 10c), we derive a relationship between $A$ and $B_{\text {Frac }}$ :

$$
A=\alpha_{1}+\alpha_{2} \exp \left(-\alpha_{3} B_{\text {Frac }}\right),
$$

where $\alpha_{1}=0.12, \alpha_{2}=0.16$, and $\alpha_{3}=10$ are empirical coefficients. The coefficients are fit in this method to bias the curve not to best fit the data weighted evenly, but to weight the curve toward data points representing simulations with more significant total mixing (more negative $M_{e}$ ). The inclusion of this $B_{\text {Frac }}$ dependence in $A$ is justified by a significantly reduced RMS error between the empirical $M_{e}$ and the LES $M_{e}$ versus using (for example) a constant mean value of $A$. The final relationship to estimate $M_{e}$ is given by

$$
M_{e} \approx \frac{\overline{w^{\prime} b_{e}^{\prime}}}{2}\left(Z_{e}\right)\left[\left(-\frac{-\overline{w^{\prime} b_{e}^{\prime}}}{\overline{w^{\prime} b_{0}^{\prime}}-\overline{w^{\prime} b_{e}^{\prime}}}\right)-A\right] .
$$




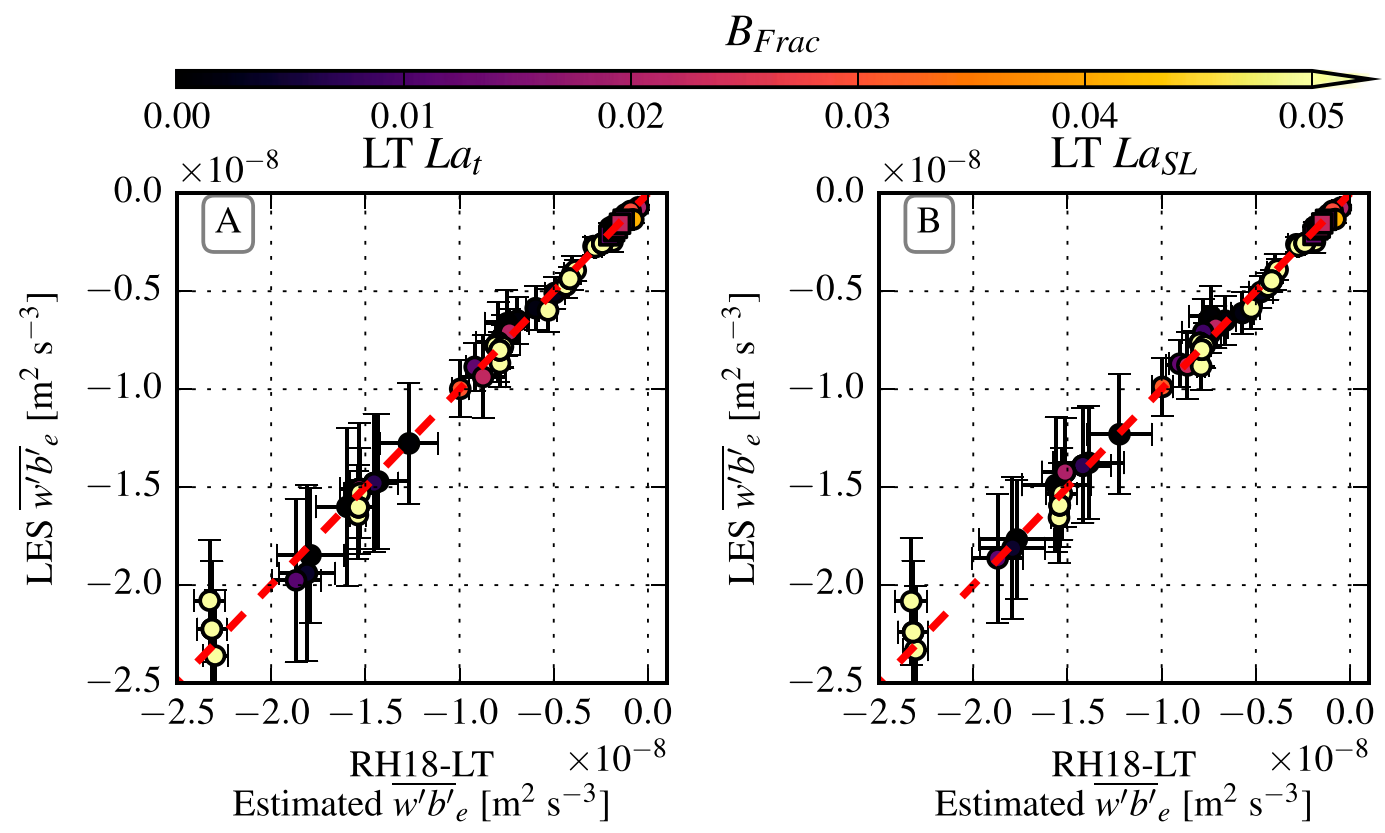

FIG. 11. Comparison of the peak entrainment buoyancy flux from the LES simulation to that inferred from Eq. (37) for (a) $\mathrm{La}_{t}$ formulation and (b) $\mathrm{La}_{\mathrm{SL}}$ formulation. The red dashed line represents the 1:1 fit, where the estimate and the derived value are the same.

Figure $10 \mathrm{~b}$ shows that this relationship accurately predicts $M_{e}$ from $\overline{w^{\prime} b_{e}^{\prime}}$ in LES.

Thus, we find that by accounting for the variability of the entrainment layer thickness $H_{e}$ through the diagnosed peak entrainment buoyancy flux $\overline{w^{\prime} b_{e}^{\prime}}$, the imposed surface buoyancy flux $\overline{w^{\prime} b_{0}^{\prime}}$, the diagnosed entrainment depth $Z_{e}$, and the diagnosed buoyancy production fraction $B_{\text {Frac }}$, we can infer the integrated entrainment buoyancy flux $M_{e}$ with a high degree of accuracy. Therefore, the LF17 entrainment buoyancy flux parameterization can be used to estimate $M_{e}$, which is investigated in the following section. In the appendix we discuss the implications of Eq. (32) for estimating the OSBL thickness.

We also note, using Eq. (36), we can use our parameterization for $M_{e}$ (section 3$)$ to predict $\overline{w^{\prime} b_{e}^{\prime}}$ :

$$
\begin{aligned}
\overline{w^{\prime} b_{e}^{\prime}} & =\frac{-a_{2}-\sqrt{a_{2}^{2}-4 a_{1} a_{3}}}{2 a_{1}}, \\
a_{1} & =\left(-Z_{e}\right)(1+A) / 2, \\
a_{2} & =Z_{e} A \overline{w^{\prime} b_{0}^{\prime}} / 2-M_{e}, \\
a_{3} & =M_{e} \overline{w^{\prime} b_{0}^{\prime}} .
\end{aligned}
$$

In Fig. 11, $\overline{w^{\prime} b_{e}^{\prime}}$ estimated from the above equation with $M_{e}$ from Eq. (20) is compared with the LES results. The consistency between Eq. (37) and LES suggests that our new parameterization for $M_{e}$ modified to include
Langmuir turbulence [Eq. (20)] is valid for predicting both $M_{e}$ and $\overline{w^{\prime} b_{e}^{\prime}}$. These relationships between $M_{e}$ and $\overline{w^{\prime} b_{e}^{\prime}}$ are valid for neutral or destabilizing surface fluxes in the absence of internally generated turbulence. Internal turbulence sources (e.g., internal wave breaking or near-inertial waves) would introduce additional parameter dependencies since additional energy sources are present. During stabilizing fluxes the quantity $\overline{w^{\prime} b_{e}^{\prime}}$ may be difficult to clearly define and could even be smaller than the surface stabilizing flux. Furthermore, penetrative solar heating can occur throughout the water column and affect the buoyancy flux profile (e.g., Pearson et al. 2015), which is not investigated here.

\section{c. Estimating $M_{e}$ following LF17}

Using Eq. (36) we can estimate $M_{e}$ from LF17 by substituting Eq. (27) for $\overline{w^{\prime} b_{e}^{\prime}}$. In Figs. 12a and 12b, we compare $\overline{w^{\prime} b_{e}^{\prime}}$ and $M_{e}$ diagnosed from LES with those predicted by the LF17 parameterization of $\overline{w^{\prime} b_{e}^{\prime}}$. We indeed find a good fit to the LES data for $M_{e}$ based on their parameterization. This result is expected because this LES data is the same that was used to derive their parameterization.

The LF17 parameterization coefficients were tuned to minimize the RMS error in predicting $\overline{w^{\prime} b_{e}^{\prime}}$, with higher weights put on the regimes where Langmuir turbulence dominates (therefore weak convection). This tuning gives a different result than tuning the coefficients in 


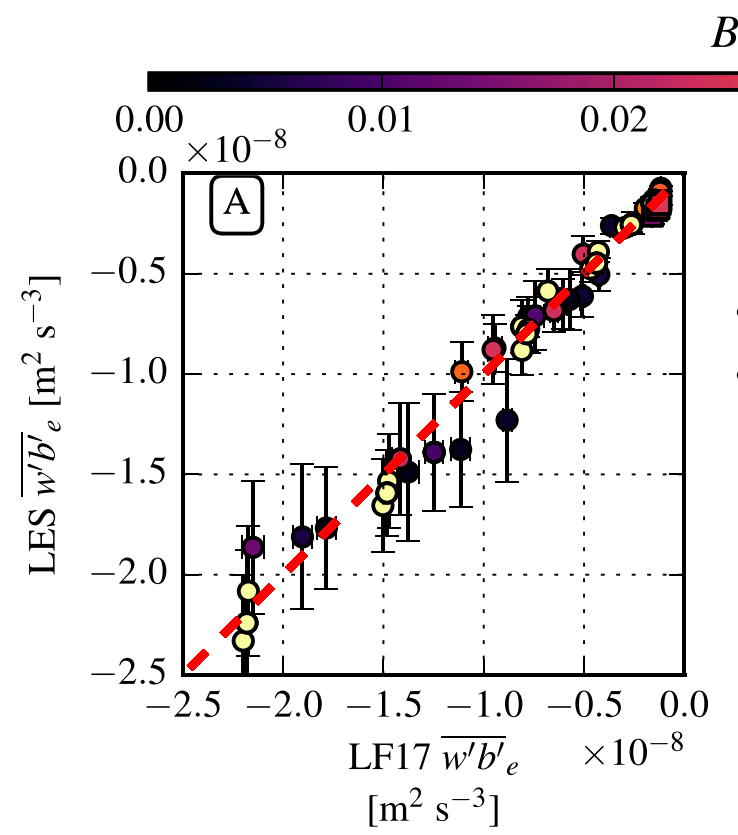

B Frac

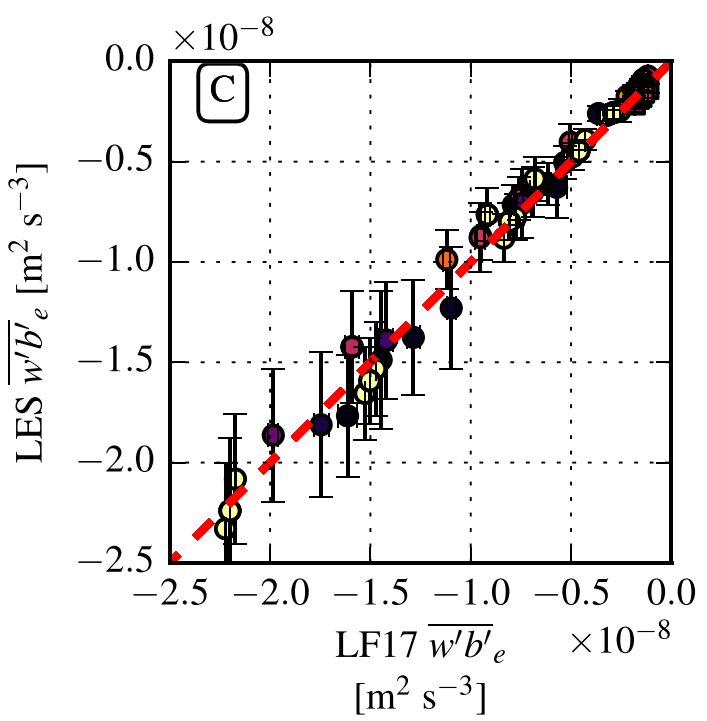

FIG. 12. (a) The peak entrainment buoyancy flux from the LES vs that predicted by LF17. (b) The computed $M_{e}$ from the LES vs the estimated $M_{e}$ using the LF17 entrainment buoyancy flux. Panels (a) and (c) and panels (b) and (d) are similar to each other, but with the optimized form of the entrainment buoyancy flux to reduce the RMS in (b) and (d).

Eq. (27) to minimize the RMS error in predicting $M_{e}$. The latter tuning strategy gives

$$
\overline{w^{\prime} b_{e}^{\prime}}=0.17 \frac{u_{*}^{3}}{H_{\mathrm{bl}}}\left(1+0.82 \mathrm{La}_{\mathrm{SL}}^{-1}\right)+0.15 \overline{w^{\prime} b_{0}^{\prime}}
$$

Employment of this relationship reduces the RMS error in predicted $M_{e}$ by approximately $25 \%$ (Figs. $12 \mathrm{~b}, \mathrm{~d}$ ). Note that we do not suggest use of Eq. (38) in place of the LF17 relationship in their modifications of KPP. Rather, we present this alternate fit here to compare $M_{e}$
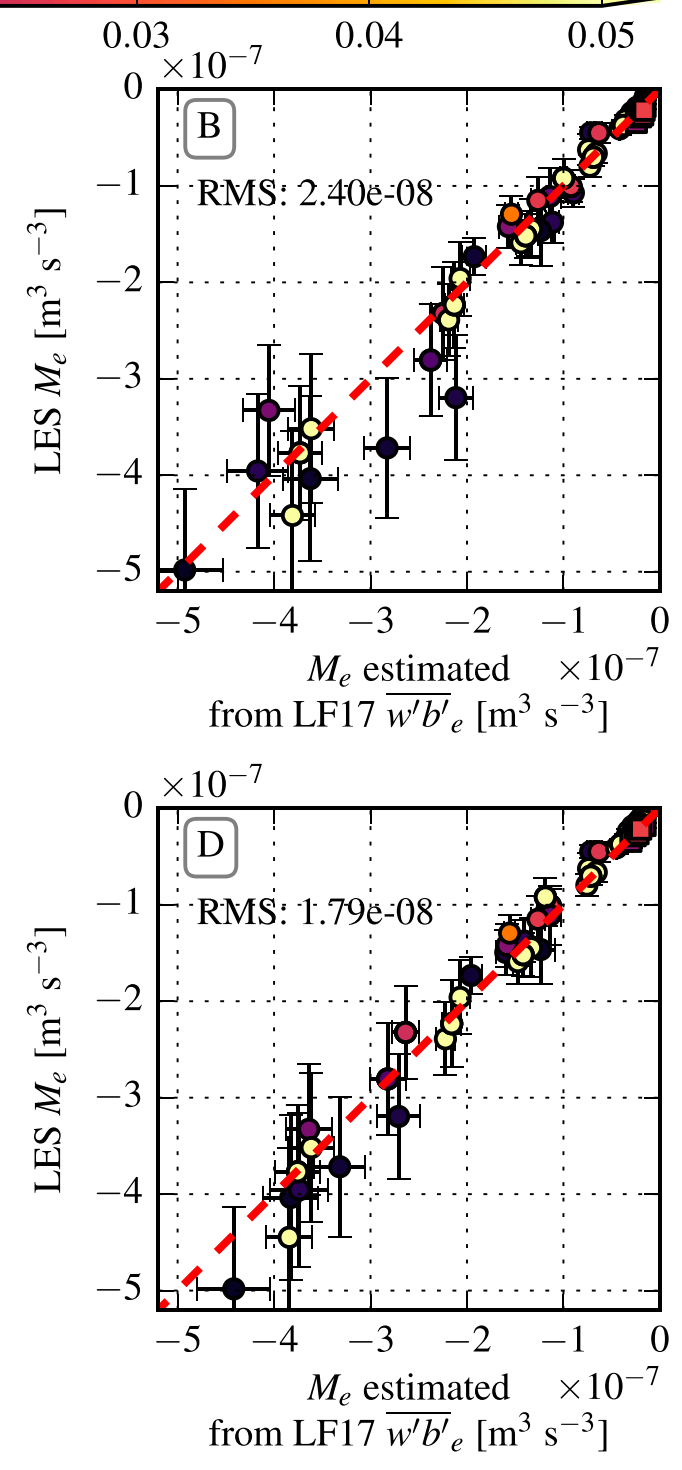

from LF17 $\overline{w^{\prime} b^{\prime}}{ }_{e}\left[\mathrm{~m}^{3} \mathrm{~s}^{-3}\right]$

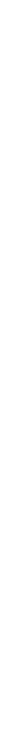


general, the approach given here and that of LF17 are compatible and provide a similar result, which is demonstrated here through comparisons of both methods with the LES results including $\overline{w^{\prime} b_{e}^{\prime}}$ (Figs. 11 and 12), $M_{e}$ (Figs. 7 and 12), and the one-dimensional simulations (Figs. 8 and 9). The result presented here obtains a lower RMS error for predicting $M_{e}$, but this result is anticipated since LF17 did not target prediction of this quantity.

The quantity $m_{*}$ as in Eq. (20) is not explicitly considered by LF17, but an analogy can be drawn from Eq. (27). If we assume that the entrainment flux is close to linear (as in Noh et al. 2003) and $M_{e} \approx 0.5 \overline{w^{\prime} b_{e}^{\prime}} H_{e}$, we can multiply Eq. (27) by $0.5 \overline{w^{\prime} b^{\prime}} H_{e}$ and find

$$
-M_{e}=\frac{H_{e}}{2}(0.17) \frac{u_{*}^{3}}{H_{\mathrm{bl}}}\left(1+0.49 \mathrm{La}_{\mathrm{SL}}^{-2}\right)+\frac{H_{e}}{2} 0.15 \overline{w^{\prime} b_{0}^{\prime}} .
$$

We can therefore speculate that $m_{*}$ of $\mathrm{RH} 18$ is performing a similar role in determining the entrainment buoyancy flux as the quantity

$$
m_{*_{\mathrm{LF} 17}} \approx 0.085 \frac{H_{e}}{H_{\mathrm{bl}}},
$$

and that $m_{*_{\mathrm{LT}}}$ introduced here is similar to

$$
m_{*_{\mathrm{LT}-\mathrm{LF} 17}} \approx 0.245 \frac{H_{e}}{H_{\mathrm{bl}}} \mathrm{La}_{\mathrm{SL}}^{-2} .
$$

In this interpretation, the RH18 formulation of $m_{*}$ can then be inferred as a parameterization of variability of the fraction $H_{e} / H_{\mathrm{bl}}$ in LF17. This is not a perfect interpretation of RH18, as the value 0.085 would also be variable in RH18, however, the dominant variability in $M_{e}$ in LF17, RH18, and this study is controlled by this ratio of the entrainment layer thickness to the boundary layer thickness. This is why both parameterizations provide similar responses in the one-dimensional simulations here, particularly as both sets of empirical coefficients are determined using the same LES. One advantage of the approach taken here for implementing within the ePBL approach is that it provides an estimate for $M_{e}$ from external forcing parameters without considering $H_{e}$. Note that, same as in Eq. (36), we are assuming simplified buoyancy flux profiles as shown in Fig. 1, which is valid based on the results of LES in quasi-equilibrium state. This simplification, for example, helps alleviate sensitivity of ePBL to vertical grid discretization, such as in section 4 a.

\section{Discussion}

In this study we found that the new parameterization for $M_{e}$ is capable of reproducing the simulated potential energy of the LES in a one-dimensional model that employs the ePBL mixing parameterization. We also found that this model is able to reproduce the effect of enhanced SST cooling by Langmuir turbulence across a range of wind and both stabilizing and destabilizing buoyancy flux conditions. Future research is needed to understand if these (and other) parameterizations remain valid over realistic conditions with diurnal cycles and penetrative solar radiation. A comparison between ePBL-LT, KPP with LF17 modifications, and many other parameterizations over realistic conditions in the global ocean is underway ( $\mathrm{Li}$ et al. 2019, manuscript submitted to J. Adv. Model. Earth Syst.).

As observed through comparing ePBL-LT with ePBL-LT-bulk, another approach that may further improve agreement of the one-dimensional model with LES is through addition of an enhancement to $K$, such as that presented by Reichl et al. (2016). However, the Reichl et al. (2016) study focused only on strongly shear-driven hurricane conditions, motivating further investigations to better parameterize all effects of Langmuir turbulence in all forcing conditions for ocean models. We have also demonstrated how the integrated buoyancy flux $\left(M_{e}\right)$ and the peak entrainment flux $\left(\overline{w^{\prime} b_{e}^{\prime}}\right)$ are related to one another due to the general similarity of the entrainment flux profile in the entrainment zone, which is a useful property to generalize future investigations into entrainment in Langmuir turbulence.

One specific future research topic is the role of nongradient mixing in convection and Langmuir turbulence and its parameterization for one-dimensional modeling. Nongradient mixing is a critical component of the KPP model in convective turbulence, which enables it to better reproduce the effect of large eddies in the OSBL. However, the one-dimensional model with KPP employed here does not reproduce the LES temperature profile, causing the inflection in the temperature profile to shoal significantly within the boundary layer, an effect of the nongradient flux representation in the model (see Fig. 8). Future research is needed to understand the vertical structure of $K$, nongradient buoyancy fluxes, and its modification by Langmuir turbulence (following Smyth et al. 2002; Noh et al. 2003; Sinha et al. 2015).

\section{Conclusions}

The present study investigates the role of Langmuir turbulence in enhancing OSBL turbulent mixing. Specifically, its effects on modifying the rate of change in potential energy through turbulent entrainment are compared to shear and convective turbulence. We first investigate parameterization of $M_{e}$ in LES cases with 
and without Langmuir turbulence and find relations capable of accurately predicting this quantity. Using the LES results of LF17 with Langmuir turbulence, we further derive a parameterization to predict $M_{e}$ in the RH18 framework in the presence of Langmuir turbulence [Eq. (20)]. This parameterization is optimized using the surface-layer averaged Langmuir number and is adjusted for the effects of convective turbulence on $M_{e}$. We then implement this new parameterization for $M_{e}$ into the ePBL parameterization framework of RH18 and demonstrate its utility for one-dimensional vertical mixing parameterization.

We also demonstrate a method to estimate the entrainment buoyancy flux $\overline{w^{\prime} b_{e}^{\prime}}$ from the integrated entrainment buoyancy flux $M_{e} \equiv \int_{Z_{e B}}^{Z_{e T}} \overline{w^{\prime} b^{\prime}} d z$, and vice versa, given the surface buoyancy flux and the entrainment depth. This relationship is useful for relating parameterizations for $\overline{w^{\prime} b_{e}^{\prime}}$ (such as LF17) to parameterizations for $M_{e}$ (this study). The relationships used to derive these quantities also yields a new method to estimate the surface boundary layer depth, $H_{\mathrm{bl}}$, that has potential utility for LES and TKE-based closure schemes (see the appendix). We conclude that the LF17 approach and the approach presented here yield generally similar results, which is not a surprising result since both are based on the same set of LES. However, we also find that implementing these relationships in time-stepping models that use ePBL and KPP can result in different behavior. This sensitivity suggests that careful consideration of potential effects of factors such as model discretization on parameterization physics is required when implementing these relationships.

An extension of this work is to explore the ePBL parameterization of RH18 with and without Langmuir turbulence in coupled climate simulations to better understand the effects of Langmuir turbulence on global mixed layer depth distributions, and to compare with the results of previous studies (Belcher et al. 2012; Fan and Griffies 2014; Noh et al. 2016; Li et al. 2016; Li and Fox-Kemper 2017). Preliminary results utilizing NOAA/GFDL's Modular Ocean Model 6 configuration for climate simulations suggest significant improvement to the OSBL depth by accounting for Langmuir turbulence in ePBL, particularly at high latitudes including the Southern Ocean. This is generally in agreement with the previous studies on the subject. Detailed experiments and analysis of such result will be presented in a follow-up manuscript.

Acknowledgments. We acknowledge many important discussions related to this work with Dr. Alistair Adcroft, Dr. Stephen Griffies, Dr. Robert Hallberg, and Dr. Baylor Fox-Kemper. We thank Dr. Andrew Shao for helpful comments on a draft of this manuscript. We also thank Dr. Luke Van Roekel and an anonymous reviewer for helpful comments that improved the quality of this manuscript. BR acknowledges support from the Cooperative Institute for Climate Science and the Cooperative Institute for Modeling the Earth's System at Princeton University and the Carbon Mitigation Initiative through Princeton's Environmental Institute at Princeton University. QL acknowledges support from NSF Grants 1258907 and 1350795 under the supervision of Dr. Baylor Fox-Kemper. This research was supported in part by the National Science Foundation under Grant NSF PHY-1748958. All the LES runs were conducted using computational resources and services at the Center for Computation and Visualization, Brown University, supported in part by the National Science Foundation EPSCoR Cooperative Agreement EPS1004057. We are grateful to Peter Sullivan for making the LES code available.

\section{APPENDIX}

\section{Estimating the Boundary Layer Depth}

There are several methods to estimate the boundary layer depth $H_{\mathrm{bl}}$ in large-eddy simulations and twoequation closure models. A reason for this ambiguity is that there is often no discrete interface between the boundary layer and the interior, and attempting to diagnose a single value within the transition layer is uncertain. Some common methods include diagnosing from maximum $N^{2}$ or a threshold criteria for a turbulent property such as the buoyancy flux, turbulent kinetic energy, or the dissipation. In this study the boundary layer depth $H_{\mathrm{bl}}$ can be estimated from the quantity $A$ [Eq. (35)] and $Z_{e}$ through relation (32). There are some potential benefits of using this method versus the other methods (e.g., arbitrariness of threshold values, $N^{2}$ peaks due to interior/hysteresis), so we will investigate the usefulness of this relation here.

One issue in employing relation (32) is that its use requires knowledge of $A$, which in turn requires knowing $B_{\text {Frac }}$, or the buoyancy production fraction in the boundary layer. Care is required while estimating the integrated buoyancy production in the OSBL $\left(G_{\mathrm{bl}}=\right.$ $\int_{Z_{e T}}^{0} \overline{w^{\prime} b^{\prime}} d z$ ), since large $\overline{w^{\prime} b^{\prime}}$ values can occur below the OSBL due to internal waves in the LES, which should not be included for the OSBL energy budget. Accurately computing the quantity $B_{\text {Frac }}$ therefore requires knowing the boundary layer thickness, which adds undesirable complexity to the algorithm required to find $H_{\mathrm{bl}}$. To eliminate this recursion, we approximate the buoyancy production term from 


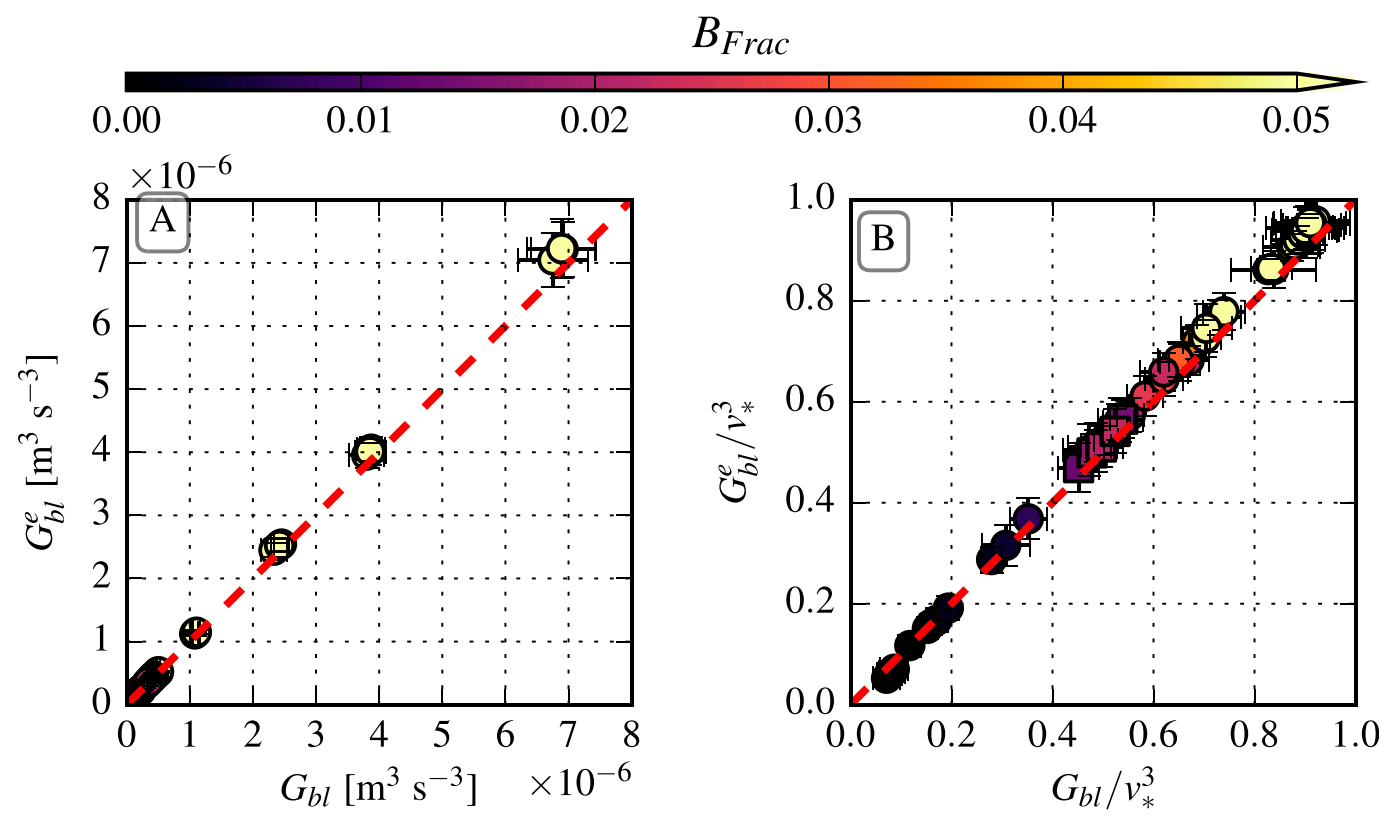

FIG. A1. Buoyancy production estimated from surface buoyancy flux and entrainment buoyancy depth vs buoyancy production integrated from LES result. (a) The dimensional data and (b) the nondimensionalized data. The red dashed line represents the 1:1 fit, where the estimate and the derived value are the same.

$$
G_{\mathrm{bl}}^{e}=\frac{\overline{w^{\prime} b_{0}^{\prime}}\left(-Z_{e}\right)}{2}\left(1+\frac{\overline{w^{\prime} b_{e}^{\prime}}}{\overline{w^{\prime} b_{0}^{\prime}}-\overline{w^{\prime} b_{e}^{\prime}}}\right) .
$$

The estimated $G_{\mathrm{bl}}^{e}$ reproduces $G_{\mathrm{bl}}$ computed from the LES result as demonstrated in both dimensional (Fig. A1a) and nondimensional (Fig. A1b) form, where

$$
v_{*}^{3}=0.4\left[0.1 u_{*}^{3}+\overline{w^{\prime} b_{0}^{\prime}}\left(-Z_{\mathrm{bl}}\right)\right]
$$

is used as a generic turbulent velocity scale to nondimensionalize the result (Fig. A1). The contributions of shear and Stokes shear to $B_{\text {Frac }}$ are estimated directly from the LES result without differentiating the OSBL contribution, since nearly all shear production occurs within the OSBL in the experiments conducted here. We thus use the quantity

$$
B_{\mathrm{Frac}} \approx \frac{G_{\mathrm{bl}}^{e}}{G_{\mathrm{bl}}^{e}+\int_{Z_{B}}^{0} \overline{w^{\prime} u_{i}^{\prime}} \partial_{z}\left(\bar{u}_{i}+\tilde{u}_{i}\right) d z}
$$

to estimate $B_{\text {Frac }}$ without prior knowledge of $H_{\mathrm{bl}}$ (where $Z_{B}$ is the bottom of the LES domain).

We compare the depth estimated from this new method and other simplified methods to that estimated using a threshold $\overline{w^{\prime} b^{\prime}}$ criteria in Fig. A2. First (Fig. A2a) we estimate the OSBL depth from the criteria for $Z_{e}$. Unsurprisingly, since we have previously shown the importance of the lower-entrainment region, this estimate underestimates the OSBL in all cases. We then (Fig. A2b) estimate the OSBL depth from the depth where $N^{2}$ reaches its maximum, another common quantity used to evaluate OSBL depth in LES. This quantity reproduces the OSBL depth compared to the $\overline{w^{\prime} b^{\prime}}$ criteria, but with a roughly constant bias of nearly $5 \mathrm{~m}$. We next explore using a constant $A=0.2$ to enhance $Z_{e}$ by a factor of 1.2 in Fig. A2c. This result has a reasonable fit to the OSBL, but shows a clear departure between 1:1 scaling varying between shear and convective cases. Finally, we use the value of $A$ predicted in this study [Eq. (35)] and estimate $H_{\mathrm{bl}} \equiv Z_{\mathrm{eB}}$ in Fig. A2d. This result shows skill for predicting the OSBL depth.

The new approach for estimating $H_{\mathrm{bl}}$ from $Z_{e}$ and $A$ is advantageous over the $\overline{w^{\prime} b^{\prime}}$ criteria (which is used as a "truth" here) because it does not use an arbitrary threshold to set the OSBL depth and it does not require algorithmic intervention for high-frequency temporal variability and internally generated $\overline{w^{\prime} b^{\prime}}$ (or similarly for TKE or dissipation criteria methods). We thus propose that this relationship can be used to evaluate an OSBL depth in a more formal manner in LES and TKE-closure parameterizations, where the definition can be ambiguous. It also may be advantageous over using $N^{2}$ in transient conditions, as $N^{2}$ may have more sensitivity to internal processes or hysteresis effects. The $Z_{e}$ may also be hard to define in transient conditions, as the $\overline{w^{\prime} b^{\prime}}$ profile might not be perfectly in the shape as illustrated 


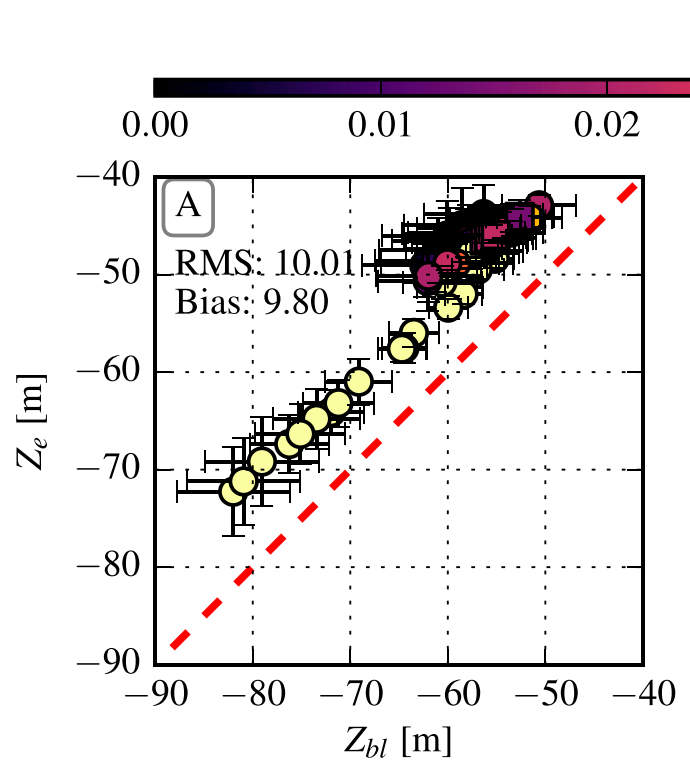

$B_{\text {Frac }}$
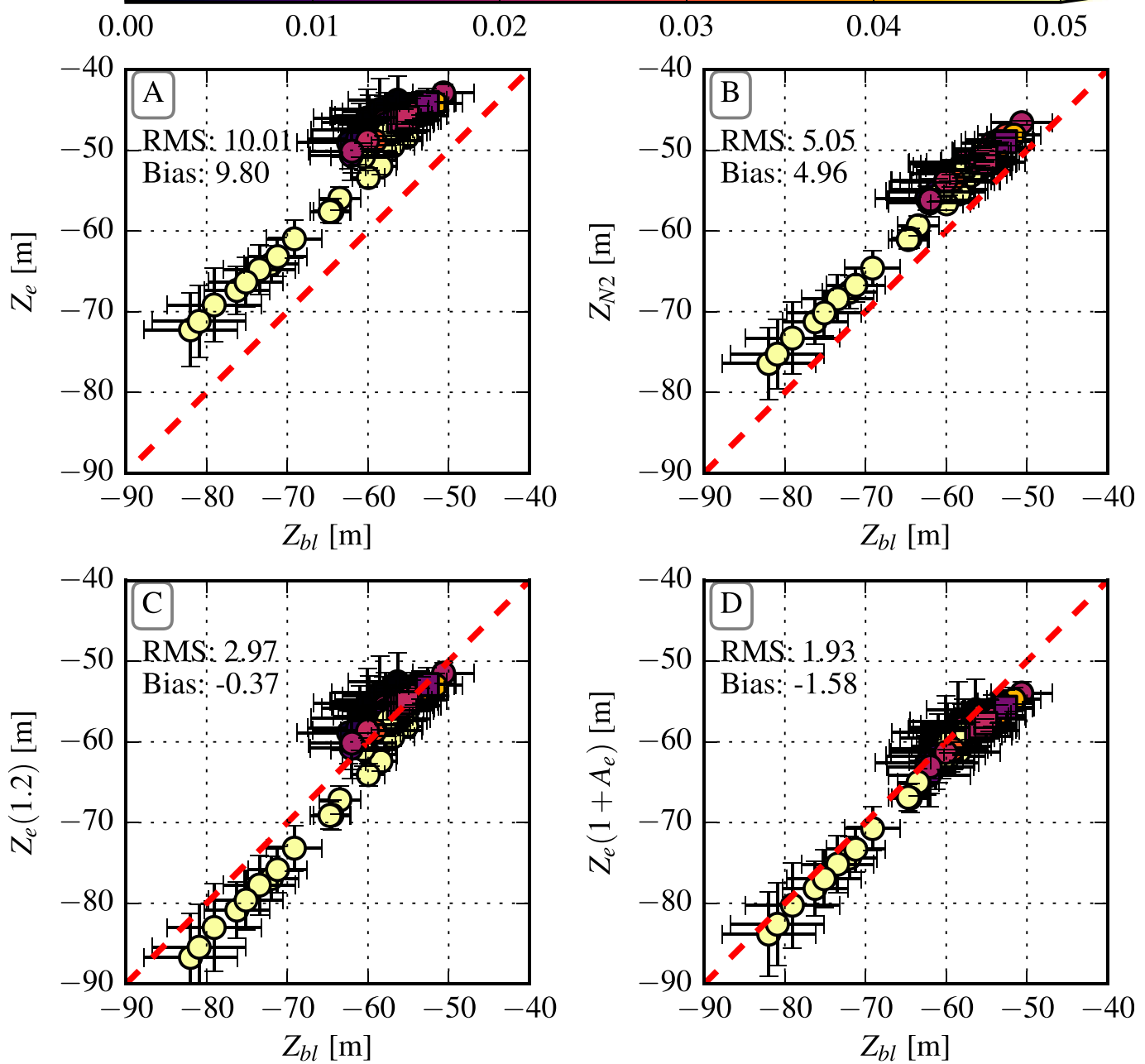

FIG. A2. Ocean surface boundary layer depth estimated from (a) the entrainment depth (minimum $\overline{w^{\prime} b^{\prime}}$ ), (b) the depth of maximum $N^{2}$, (c) the entrainment depth multiplied by a constant enhancement, and (d) the entrainment depth multiplied by coefficient $A$ from this study vs $Z_{\mathrm{bl}}$ computed from the LES simulations using a $\overline{w^{\prime} b^{\prime}}$ threshold criteria. The red dashed line represents the 1:1 fit, where the estimate and the derived value are the same.

in Fig. 1, however, its response time to the active turbulence is shorter than that of the profile of $N^{2}$.

This result requires further investigation in future studies to ensure robustness and to explore how applicable this method is for isolating OSBL from internally generated turbulence sources in nonidealized onedimensional simulations. Such investigations are specifically needed to adapt this method for use in global ocean simulations that utilize TKE closure type mixing parameterizations, particularly when internal turbulence generation regions interact with the entrainment layer and the entrainment layer is therefore no longer entirely surface driven. Such scenarios would complicate evaluation of the shear-production contribution, since any internal shear layers should be neglected. Furthermore, the diagnosed quantity $\overline{w^{\prime} b_{e}^{\prime}}$ may also be modified by the internal turbulence. Such issues more fundamentally expose the unnatural separation of the surface boundary layer and turbulence below the base of the boundary layer, which is the primary source of ambiguity in the definition of a boundary layer depth to begin with. However, based on this study this boundary layer depth calculation shows potential usefulness outside of the application for which it was derived here.

\section{REFERENCES}

Adcroft, A., R. W. Hallberg, and the MOM6 team, 2018: MOM6 model source and documentation. https://github.com/ NOAA-GFDL/MOM6-examples/wiki. 
Agrawal, Y. C., E. A. Terray, M. A. Donelan, P. A. Hwang, A. J. Williams III, W. M. Drennan, K. K. Kahma, and S. A. Kitaigorodskii, 1992: Enhanced dissipation of kinetic energy beneath surface waves. Nature, 359, 219-220, https://doi.org/ 10.1038/359219a0.

Anis, A., and J. N. Moum, 1995: Surface wave-turbulence interactions: Scaling $\varepsilon(z)$ near the sea surface. J. Phys. Oceanogr., 25, 2025-2045, https://doi.org/10.1175/1520-0485(1995) 025<2025:SWISNT>2.0.CO;2.

Axell, L. B., 2002: Wind-driven internal waves and Langmuir circulations in a numerical ocean model of the southern Baltic Sea. J. Geophys. Res., 107, 3204, https://doi.org/10.1029/2001JC000922.

Belcher, S. E., and Coauthors, 2012: A global perspective on Langmuir turbulence in the ocean surface boundary layer. Geophys. Res. Lett., 39, L18605, https://doi.org/10.1029/ 2012 GL052932.

Caughey, S. J., and S. G. Palmer, 1979: Some aspects of turbulence structure through the depth of the convective boundary layer. Quart. J. Roy. Meteor. Soc., 105, 811-827, https://doi.org/ 10.1002/qj.49710544606.

Craik, A. D. D., and S. Leibovich, 1976: A rational model for Langmuir circulations. J. Fluid Mech., 73, 401-426, https:// doi.org/10.1017/S0022112076001420.

D'Asaro, E. A., 2014: Turbulence in the upper-ocean mixed layer. Annu. Rev. Mar. Sci., 6, 101-115, https://doi.org/10.1146/ annurev-marine-010213-135138.

D’Alessio, S. J. D., K. Abdella, and N. A. McFarlane, 1998: A new second-order turbulence closure scheme for modeling the oceanic mixed layer. J. Phys. Oceanogr., 28, 16241641, https://doi.org/10.1175/1520-0485(1998)028<1624: ANSOTC $>2.0 . \mathrm{CO} ; 2$.

Donelan, M. A., J. Hamilton, and W. H. Hui, 1985: Directional spectra of wind-generated waves. Philos. Trans. Roy. Soc. London, 315A, 509-562, https://doi.org/10.1098/rsta.1985.0054.

Fan, Y., and S. M. Griffies, 2014: Impacts of parameterized Langmuir turbulence and nonbreaking wave mixing in global climate simulations. J. Climate, 27, 4752-4775, https://doi.org/ 10.1175/JCLI-D-13-00583.1.

Gerbi, G. P., J. H. Trowbridge, E. A. Terray, A. J. Plueddemann, and T. Kukulka, 2009: Observations of turbulence in the ocean surface boundary layer: Energetics and transport. J. Phys. Oceanogr., 39, 1077-1096, https:// doi.org/10.1175/2008JPO4044.1.

Grant, A. L., and S. E. Belcher, 2009: Characteristics of Langmuir turbulence in the ocean mixed layer. J. Phys. Oceanogr., 39, 1871-1887, https://doi.org/10.1175/2009JPO4119.1.

Harcourt, R. R., 2013: A second-moment closure model of Langmuir turbulence. J. Phys. Oceanogr., 43, 673-697, https:// doi.org/10.1175/JPO-D-12-0105.1.

_ 2015: An improved second-moment closure model of Langmuir turbulence. J. Phys. Oceanogr., 45, 84-103, https:// doi.org/10.1175/JPO-D-14-0046.1.

_- and E. A. D'Asaro, 2008: Large-eddy simulation of Langmuir turbulence in pure wind seas. J. Phys. Oceanogr., 38, 15421562, https://doi.org/10.1175/2007JPO3842.1.

Hewitt, H. T., and Coauthors, 2017: Will high-resolution global ocean models benefit coupled predictions on short-range to climate timescales? Ocean Modell., 120, 120-136, https:// doi.org/10.1016/j.ocemod.2017.11.002.

Hoecker-Martínez, M. S., W. D. Smyth, and E. D. Skyllingstad, 2016: Oceanic turbulent energy budget using large-eddy simulation of a wind event during DYNAMO.J. Phys. Oceanogr., 46, 827-840, https://doi.org/10.1175/JPO-D-15-0057.1.
Jackson, L., R. Hallberg, and S. Legg, 2008: A parameterization of shear-driven turbulence for ocean climate models. J. Phys. Oceanogr., 38, 1033-1053, https://doi.org/10.1175/ 2007JPO3779.1.

Kantha, L. H., and C. A. Clayson, 2004: On the effect of surface gravity waves on mixing in the oceanic mixed layer. Ocean Modell., 6, 101-124, https://doi.org/10.1016/S1463-5003(02)00062-8.

Kukulka, T., and R. R. Harcourt, 2017: Influence of Stokes drift decay scale on Langmuir turbulence. J. Phys. Oceanogr., 47, 1637-1656, https://doi.org/10.1175/JPO-D-16-0244.1.

_ A. J. Plueddemann, J. H. Trowbridge, and P. P. Sullivan, 2009: Significance of Langmuir circulation in upper ocean mixing: Comparison of observations and simulations. Geophys. Res. Lett., 36, L10603, https://doi.org/10.1029/2009GL037620.

$-, \ldots,-$, and $\_, 2010$ : Rapid mixed layer deepening by the combination of Langmuir and shear instabilities: A case study. J. Phys. Oceanogr., 40, 2381-2400, https://doi.org/ 10.1175/2010JPO4403.1.

Large, W. G., J. C. McWilliams, and S. C. Doney, 1994: Oceanic vertical mixing: A review and a model with a nonlocal boundary layer parameterization. Rev. Geophys., 32, 363-403, https://doi.org/10.1029/94RG01872.

Li, M., C. Garrett, and E. Skyllingstad, 2005: A regime diagram for classifying turbulent large eddies in the upper ocean. Deep-Sea Res. I, 52, 259-278, https://doi.org/10.1016/j.dsr.2004.09.004.

Li, Q., and B. Fox-Kemper, 2017: Assessing the effects of Langmuir turbulence on the entrainment buoyancy flux in the ocean surface boundary layer. J. Phys. Oceanogr., 47, 2863-2886, https://doi.org/10.1175/JPO-D-17-0085.1.

_ A. Webb, B. Fox-Kemper, A. Craig, G. Danabasoglu, W. G. Large, and M. Vertenstein, 2016: Langmuir mixing effects on global climate: WAVEWATCH III in CESM. Ocean Modell., 103, 145-160, https://doi.org/10.1016/j.ocemod.2015.07.020.

McWilliams, J. C., and P. P. Sullivan, 2000: Vertical mixing by Langmuir circulations. Spill Sci. Technol. Bull., 6, 225-237, https://doi.org/10.1016/S1353-2561(01)00041-X.

,-- , and C. H. Moeng, 1997: Langmuir turbulence in the ocean. J. Fluid Mech., 334, 1-30, https://doi.org/10.1017/ S0022112096004375.

_ E. Euckle, J.-H. Liang, and P. P. Sullivan, 2012: The wavy Ekman layer: Langmuir circulations, breaking waves, and Reynolds stress. J. Phys. Oceanogr., 42, 1793-1816, https:// doi.org/10.1175/JPO-D-12-07.1.

_ _ _ - J. Liang, and P. P. Sullivan, 2014: Langmuir turbulence in swell. J. Phys. Oceanogr., 44, 870-890, https://doi.org/ 10.1175/JPO-D-13-0122.1.

Melville, W. K., 1996: The role of surface-wave breaking in air-sea interaction. Annu. Rev. Fluid Mech., 28, 279-321, https:// doi.org/10.1146/annurev.fl.28.010196.001431.

Noh, Y., W. G. Cheon, S. Y. Hong, and S. Raasch, 2003: Improvement of the k-profile model for the planetary boundary layer based on large eddy simulation data. Bound.-Layer Meteor., 107, 401-427, https://doi.org/10.1023/A:1022146015946.

_ S. Min, and S. Raasch, 2004: Large eddy simulation of the ocean mixed layer: The effects of wave breaking and Langmuir circulation. J. Phys. Oceanogr., 34, 720-735, https://oi.org/10.1175/ 1520-0485(2004)034<0720:LESOTO > 2.0.CO;2.

—, H. Ok, E. Lee, T. Toyoda, and N. Hirose, 2016: Parameterization of Langmuir circulation in the ocean mixed layer model using les and its application to the OGCM. J. Phys. Oceanogr., 46, 57-78, https://doi.org/10.1175/JPO-D-14-0137.1.

Pearson, B. C., A. L. M. Grant, J. A. Polton, and S. E. Belcher, 2015: Langmuir turbulence and surface heating in the ocean 
surface boundary layer. J. Phys. Oceanogr., 45, 2897-2911, https://doi.org/10.1175/JPO-D-15-0018.1.

Pizzo, N. E., L. Deike, and W. K. Melville, 2016: Current generation by deep-water breaking waves. J. Fluid Mech., 803, 275291, https://doi.org/10.1017/jfm.2016.469.

Polton, J. A., and S. E. Belcher, 2007: Langmuir turbulence and deeply penetrating jets in an unstratified mixed layer. J. Geophys. Res., 112, C09020, https://doi.org/10.1029/2007JC004205.

Reichl, B. G., and R. Hallberg, 2018: A simplified energetics based planetary boundary layer (ePBL) approach for ocean climate simulations. Ocean Modell., 132, 112-129, https://doi.org/ 10.1016/j.ocemod.2018.10.004.

—_, D. Wang, T. Hara, I. Ginis, and T. Kukulka, 2016: Langmuir turbulence parameterization in tropical cyclone conditions. J. Phys. Oceanogr., 46, 863-886, https://doi.org/ 10.1175/JPO-D-15-0106.1.

Sinha, N., A. E. Tejada-Martínez, C. Akan, and C. E. Grosch, 2015: Toward a K-profile parameterization of Langmuir turbulence in shallow coastal shelves. J. Phys. Oceanogr., 45, 2869-2895, https://doi.org/10.1175/JPO-D-14-0158.1.

Skyllingstad, E. D., and D. W. Denbo, 1995: An ocean large-eddy simulation of Langmuir circulations and convection in the surface mixed layer. J. Geophys. Res., 100, 8501-8522, https:// doi.org/10.1029/94JC03202.

Smith, J. A., 1992: Observed growth of Langmuir circulation. J. Geophys. Res., 97, 5651-5664, https://doi.org/10.1029/ 91JC03118.

Smyth, W. D., E. D. Skyllingstad, G. B. Crawford, and H. W. Wijesekera, 2002: Nonlocal fluxes and Stokes drift effects in the K-profile parameterization. Ocean Dyn., 52, 104-115, https://doi.org/10.1007/s10236-002-0012-9.
Sullivan, P. P., J. C. McWilliams, and W. K. Melville, 2007: Surface gravity wave effects in the oceanic boundary layer: Large-eddy simulation with vortex force and stochastic breakers. J. Fluid Mech., 593, 405-452, https:// doi.org/10.1017/S002211200700897X.

Teixeira, M. A. C., and S. E. Belcher, 2002: On the distortion of turbulence by a progressive surface wave. J. Fluid Mech., 458, 229-267, https://doi.org/10.1017/S0022112002007838.

Thomson, J., S. Schwendeman, S. F. Zippel, S. Moghimi, and J. Gemmrich, 2016: Wave-breaking turbulence in the ocean surface layer. J. Phys. Oceanogr., 46, 1857-1870, https:// doi.org/10.1175/JPO-D-15-0130.1.

Van Den Bremer, T. S., and O. Breivik, 2017: Stokes drift. Philos. Trans. Roy. Soc. London, 376A, 1-23, https://doi.org/10.1098/ RSTA.2017.0104.

Van Roekel, L. P., B. Fox-Kemper, P. P. Sullivan, P. E. Hamlington, and S. R. Haney, 2012: The form and orientation of Langmuir cells for misaligned winds and waves. J. Geophys. Res., 117, C05001, https://doi.org/10.1029/2011JC007516.

_ , and Coauthors, 2018: The KPP boundary layer scheme for the ocean: Revisiting its formulation and benchmarking one-dimensional simulations relative to LES. J. $A d v$. Model. Earth Syst., 10, 2647-2684, https://doi.org/10.1029/ 2018MS001336.

vanZanten, M. C., P. G. Duynkerke, and J. W. M. Cuijpers, 1999: Entrainment parameterization in convective boundary layers. J. Atmos. Sci., 56, 813-828, https://doi.org/10.1175/15200469(1999)056<0813:EPICBL>2.0.CO;2.

Weller, R. A., and J. F. Price, 1988: Langmuir circulations within the oceanic mixed layer. Deep-Sea Res., 35, 711-747, https:// doi.org/10.1016/0198-0149(88)90027-1. 